ARTICLE

\title{
The secreted protease Adamts18 links hormone action to activation of the mammary stem cell niche
}

Dalya Ataca (1) 1,6, Patrick Aouad ${ }^{1,6}$, Céline Constantin 1,6, Csaba Laszlo1, Manfred Beleut (1) 1,4, Marie Shamseddin (1) 1,2, Renuga Devi Rajaram', Rachel Jeitziner ${ }^{1,5}$, Timothy J. Mead (1) 3, Marian Caikovski ${ }^{1,5}$, Philipp Bucher ${ }^{1}$, Giovanna Ambrosini ${ }^{1}$, Suneel S. Apte ${ }^{3} \&$ Cathrin Brisken (10) ${ }^{1 凶}$

Estrogens and progesterone control breast development and carcinogenesis via their cognate receptors expressed in a subset of luminal cells in the mammary epithelium. How they control the extracellular matrix, important to breast physiology and tumorigenesis, remains unclear. Here we report that both hormones induce the secreted protease Adamts18 in myoepithelial cells by controlling Wnt4 expression with consequent paracrine canonical Wnt signaling activation. Adamts18 is required for stem cell activation, has multiple binding partners in the basement membrane and interacts genetically with the basal membranespecific proteoglycan, Col18a1, pointing to the basement membrane as part of the stem cell niche. In vitro, ADAMTS18 cleaves fibronectin; in vivo, Adamts18 deletion causes increased collagen deposition during puberty, which results in impaired Hippo signaling and reduced Fgfr2 expression both of which control stem cell function. Thus, Adamts18 links luminal hormone receptor signaling to basement membrane remodeling and stem cell activation.

\footnotetext{
${ }^{1}$ Ecole Polytechnique Fédérale de Lausanne, Station 19, CH-1015 Lausanne, Switzerland. ${ }^{2}$ Wellcome Sanger Institute, Wellcome Genome Campus, Hinxton, Cambridge CB10 1SA, UK. ${ }^{3}$ Department of Biomedical Engineering-ND2O, Cleveland Clinic Lerner Research Institute, 9500 Euclid Ave., Cleveland, OH 44195, USA. ${ }^{4}$ Present address: Medoderm GmbH, Robert Koch-Straße 50 D, 55129 Mainz, Germany. ${ }^{5}$ Present address: Swiss Institute of Bioinformatics, Agora Swiss Cancer Center Leman, Rue du Bugnon 25a, 1015 Lausanne, Switzerland. ${ }^{6}$ These authors contributed equally: Dalya Ataca, Patrick Aouad, Céline Constantin. ${ }^{凶}$ email: cathrin.brisken@epfl.ch
} 
T he breast is the only organ to develop mostly after birth. Milk ducts arborize from the nipple and grow into a specialized subcutaneous stroma called the mammary fat pad in mice. The ductal wall comprises a bi-layered epithelium with the inner luminal cells and outer myoepithelial cells. The epithelium is separated from the stroma by specialized extracellular matrix (ECM), the basement membrane (BM). The ovarian hormones, estrogens and progesterone, are key drivers of mammary gland development and also influence breast carcinogenesis ${ }^{1}$. Both estrogen receptor a (ER) and progesterone receptor (PR) are members of the nuclear receptor family and are readily detected by immunohistochemistry (IHC) in a subset of luminal cells ${ }^{1}$. Activation of hormone receptor signaling in cells with high hormone receptor expression, termed sensor cells ${ }^{2}$, triggers the expression of paracrine factors such as amphiregulin and Rankl, which are required for mammary epithelial cell proliferation ${ }^{3,4}$ as well as Wnt4 and Cxcl12, which activate stem/progenitor cells ${ }^{5,6}$. Mammary stem and progenitor cells have been identified and characterized based on cell surface markers and functional assays ${ }^{7}$. However, the precise cellular and biochemical components of the stem cell niche and its endocrine regulation remain poorly defined.

Evidence has been provided that mammary ECM can reprogram non-mammary cells to form mammary glands ${ }^{8,9}$, suggesting that it contains critical cues for epithelial development. Hedgehog signaling acts via Gli2 downstream of growth hormone receptor signaling in fibroblasts to trigger changes in paracrine signaling and ECM proteins that affect stem cell function ${ }^{10}$. This suggests that stromal fibroblasts are part of the niche under direct endocrine control by growth hormone. Stromal changes accompany different morphogenic processes induced by epithelial hormone signaling and are a hallmark of breast carcinogenesis. Indeed, high radiographic density, which reflects an increase in fibrillar collagen content in the breast stroma, is the single most important risk factor for breast cancer and correlates with progesterone exposure ${ }^{11,12}$. How ECM and stroma are controlled by the major endocrine drivers of breast development and carcinogenesis, epithelial ER and PR signaling, remains elusive.

ADAMTS18 is an orphan member of the A Disintegrin-like And Metalloproteinase domain with ThromboSpondin type 1 Motifs (ADAMTS) family of secreted $\mathrm{Zn}$-dependent metalloproteinases $^{13}$ that comprises 19 members $^{14,15}$. Like other zinc metalloproteinases, ADAMTS catalytic activity depends on zinc ion binding within the active site; unique to ADAMTSs is an ancillary domain containing thrombospondin type 1 repeats ${ }^{16}$. ADAMTS proteases are synthesized as precursors with an $\mathrm{N}$-terminal propeptide, which is excised by pro-protein convertases such as furin ${ }^{14}$. Some ADAMTSs process ECM components such as fibrillar collagens, while others are implicated in turnover of the chondroitin sulfate proteoglycans aggrecan and versican ${ }^{14}$, and ADAMTS13 uniquely cleaves von-Willebrand factor to maturity ${ }^{17}$. We have previously reported that Adamts18 is required for eye, lung and female reproductive tract and kidney development in the mouse $^{18}$. It is highly homologous to Adamts16, which has a role in renal development and fertility 19,20 and can cleave fibronectin ${ }^{21}$. Here, we show that Adamts18 provides a mechanistic link between epithelial steroid hormone receptor signaling and changes in the $\mathrm{ECM}$, in particular the BM, that regulate mammary epithelial stemness.

\section{Results}

Adamts18 expression is driven by the $P R / W n t 4$ axis. To elucidate the mechanisms, by which PR signaling in luminal mammary epithelial cells may elicit ECM changes, we sought genes induced in vivo by progesterone treatment ${ }^{22,23}$ that fulfilled two criteria: (1) They encoded secretory proteins and (2) They showed delayed induction by progesterone as expected of any indirect PR target which is expressed by myoepithelial cells and can hence directly interact with the BM. Adamts 18 induction was detected at 16 hours (h) and $78 \mathrm{~h}$ but not at $4 \mathrm{~h}^{22}$ and at $24 \mathrm{~h}$ but not $8 \mathrm{~h}$ following progesterone stimulation ${ }^{23}$. RT-PCR analysis of fluorescence activated cell sorting (FACS)-sorted cells from adult mammary glands showed a 7-fold enrichment of Adamts18 mRNA in myoepithelial ( $\mathrm{Lin}^{-} \mathrm{CD} 24^{+} \mathrm{CD}^{-} 9 \mathrm{f}^{+}$) over luminal ( Lin $^{-} \mathrm{CD} 24^{+} \mathrm{CD}^{-} \mathrm{f}^{-}$) cells (Fig. 1a), in line with recent single cell RNA sequencing data 24,25 , confirming expression in myoepithelial cells.

Analysis of Adamts18 transcript levels at different stages of mammary gland development revealed low prepubertal expression that increased 2.7, 7- and 8.6-fold in 4-, 6- and 8-week-old females, respectively; expression rose further during pregnancy with a peak at mid-pregnancy day10.5/12.5 (Fig. 1b). RNAscope in situ hybridization for Adamts 18 transcripts combined with immunofluorescence (IF) for the myoepithelial marker $\alpha$-smooth muscle actin (Sma) confirmed myoepithelium-specific expression of Adamts18 in pubertal and adult mammary ducts (Fig. 1c, d). The increased Adamts18 expression during pregnancy was not attributable to generalized but rather to myoepithelium-specific upregulation of expression (Fig. 1e). Thus, Adamts18 expression in the mammary epithelium is developmentally regulated, and its mRNA is enriched in myoepithelial cells, making it an attractive candidate to mediate ECM changes downstream of epithelial hormone action.

Next, we tested whether endocrine factors contribute to developmental Adamts18 expression. First, we mimicked pubertal estrogen stimulation by injecting ovariectomized 21 -day-old mice with 17 - $\beta$-estradiol. Within $18 \mathrm{~h}$ of injection, Adamts 18 transcript levels in extracts from total mammary glands increased 1.76-fold (Fig. 1f). Second, we asked whether changes in progesterone levels as they occur during estrous cycles affect Adamts18 transcript levels and obtained mammary gland extracts from mice in estrus and diestrus. Progesterone plasma levels determined by liquid chromatography-mass spectrometry were on average 2.8 -fold higher in diestrus than in estrus (Fig. 1g); Adamts18 transcript levels in the mammary glands were 1.6-fold higher in diestrous over estrous (Fig. 1h). Thus, physiological Adamts18 expression correlates with plasma progesterone levels, suggesting that it is progesterone-responsive. The subtle increases in transcript levels are consistent with myoepithelial cells representing a minor fraction of the mammary cell types and hence of the total RNA in the whole tissue extracts we analyzed.

To determine whether epithelium-intrinsic PR signaling is required for Adamts 18 mRNA expression, mammary epithelia from $W T . E G F P^{+}$and $P^{-/-} . E G F P^{+}$mice were grafted to contralateral fat pads of $W T$ recipients surgically cleared of the endogenous epithelium and allowed to grow out for six weeks. At sacrifice, reconstitution was validated by fluorescence stereomicroscopy of the engrafted glands. Adamts 18 transcript levels in the mammary glands successfully reconstituted with $P R^{-/-}$ epithelium were on average $27 \%$ of those in the contralateral controls (Fig. 1i). Thus, epithelial $P R$ expression is required for Adamts18 mRNA expression.

Wnt4 is a plausible candidate to induce Adamts18 expression in myoepithelial cells because it is a PR target ${ }^{26}$ transcribed exclusively in PR + luminal cells ${ }^{6}$ and activates canonical Wnt signaling in the myoepithelial cells ${ }^{6}$, which express Adamts18. We analyzed expression of various Wnt signaling components expressed in the mammary epithelium by RT-PCR in contralateral glands engrafted with WT.EGFP ${ }^{+}$and $\mathrm{PR}^{-/-} . E G F P^{+}$ mammary epithelia. Among the Wnt genes, only Wnt4 transcript levels were significantly lower in the mutant grafts, furthermore 
a

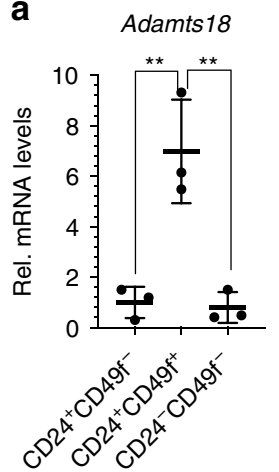

b

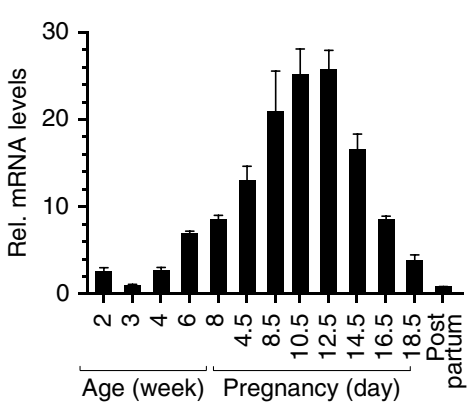

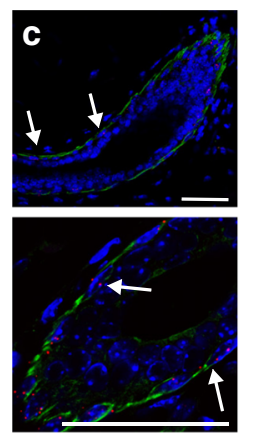
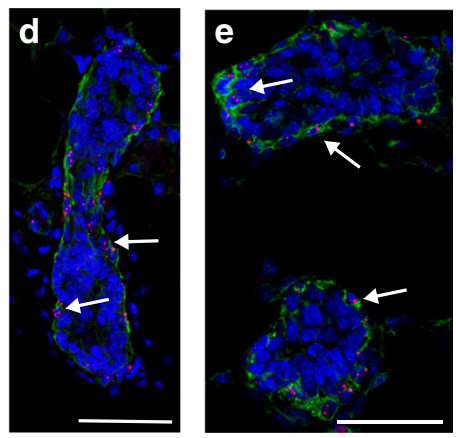

$f$

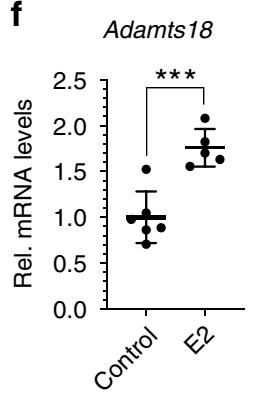

g

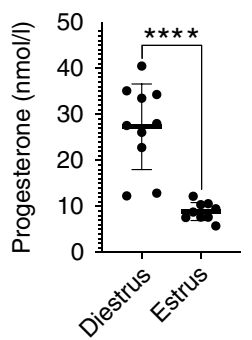

h

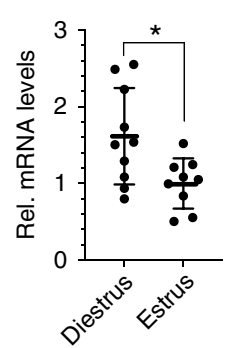

Adamts 18

j
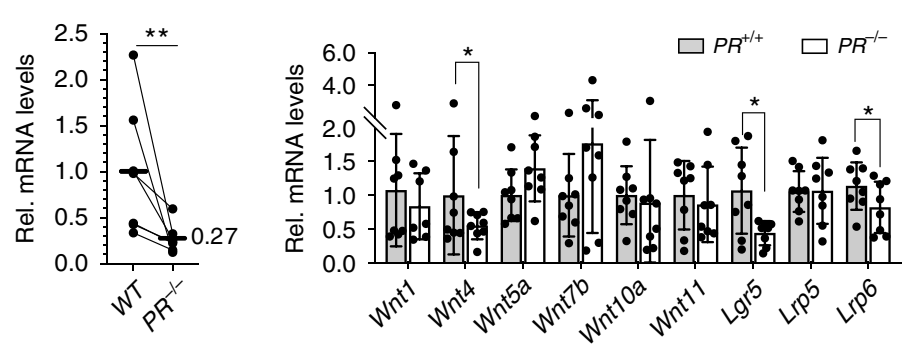

k

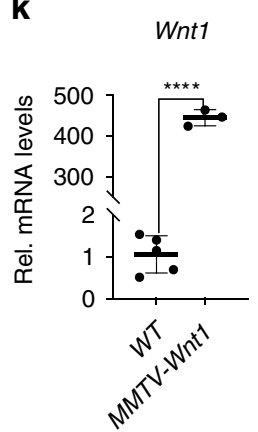

Axin2

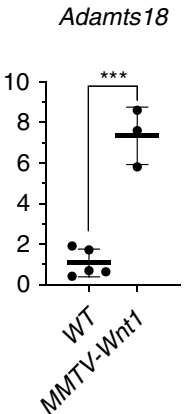

I

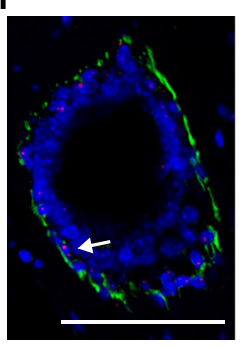

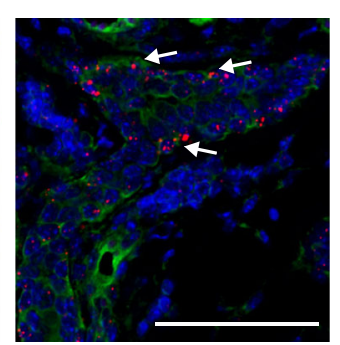

m

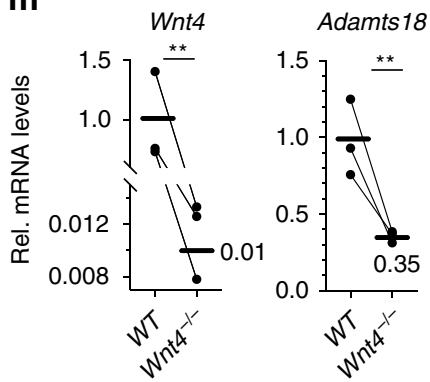

Fig. 1 Adamts18 expression in the mouse mammary gland. a Dot plot showing Adamts18 mRNA expression normalized to Hprt in FACS-sorted CD24+ CD49f- (luminal), CD24+CD49f + (myoepithelial) and CD24-CD49f- (stromal) cells. Data represent mean \pm SD from $n=3$ independent experiments. Student $t$-test, two-tailed. b Bar plot showing Adamts18 mRNA levels normalized to Hprt in mammary glands at different developmental stages. Each bar represents pool of 3 mice, mean \pm SD for technical replicates. c-e Representative micrographs showing Adamts18 mRNA localization in mouse mammary gland during puberty (c), adulthood (d) and pregnancy day 12.5 (e). Red dots represent Adamts18 in situ hybridization signal, green: $\alpha$-Sma, blue: DAPI, arrows show myoepithelial cells; scale bar, $50 \mu \mathrm{m}$. f Relative Adamts 18 transcript levels normalized to Krt5 in mammary glands from 6 control and 5 E2treated mice. Data represent mean \pm SD, unpaired Student $t$-test, two-tailed. $\mathbf{g}$ Dot plot showing plasma progesterone levels determined by LC/MS during diestrus $(n=10)$ or estrus $(n=9)$. Data represent mean \pm SD, Student $t$-test, two-tailed. $\mathbf{h}$ Dot plot showing Adamts18 mRNA levels normalized to Krt5 in mammary glands from mice shown in $\mathbf{g}$. Data represent mean \pm SD, Student $t$-test, two-tailed. i Dot plot showing Adamts18 mRNA normalized to Hprt in 6 contralateral mammary glands transplanted with WT.EGFP+ or $P R^{-/-}$.EGFP+ epithelium. $\mathbf{j}$ Bar graph showing relative transcript expression of different Wnt signaling components normalized to Hprt in contralateral glands of 8 mice transplanted with WT.EGFP+ and PR ${ }^{-/-}$.EGFP+ epithelia. Each data point represents one gland, mean $\pm S D$, paired Student $t$-test, two-tailed. k Dot plots showing relative transcript levels of Wnt1, Axin2 and Adamts18 normalized to Hprt in mammary glands from 5 WT and 3 MMTV-Wnt1 virgin mice. Data represent mean \pm SD, Student $t$-test, two-tailed. I Representative micrographs of Adamts18 mRNA localization, (red) dots, in mammary glands from 3 WT and 3 MMTV-Wnt1 females, $\alpha$-Sma (green) and DAPI (blue); arrows show myoepithelial cells. Scale bar, $50 \mu \mathrm{m}$. $\mathbf{m}$ Dot plots showing mRNA levels of Wnt4 and Adamts 18 normalized to Hprt in contralateral glands of 3 mice transplanted with WT.EGFP+ and Wnt4-/-.EGFP+ epithelia harvested at 8.5-day of pregnancy. ${ }^{\star} p<0.05 ;{ }^{\star \star} p<0.01 ;{ }^{\star \star \star} p<0.001 ;{ }^{\star \star \star \star \star} p<0.0001$.

the transcript levels of the stem cell marker Lgr5 and the Wnt coreceptor Lpr6 were decreased (Fig. 1j). Consistent with canonical Wnt signaling activation downstream of PR/Wnt4 controlling Adamts 18 expression, TCF4 binding sites were reported in the Adamts 18 promoter by ChIP-seq analysis ${ }^{27}$. To assess whether canonical Wnt signaling controls Adamts 18 expression in vivo, we analyzed Adamts 18 expression in mammary glands with hyperactive canonical Wnt signaling in the myoepithelium ${ }^{6}$ due to the presence of an $M M T V-W n t 1$ transgene ${ }^{28}$. Ectopic
Wnt1 expression was readily detected in transgenic glands and expression of the canonical Wnt signaling target, Axin2, was increased 5-fold over the non-transgenic control while Adamts18 mRNA levels were increased 7-fold (Fig. 1k). RNAscope for Adamts18 transcripts combined with IF for Sma showed the increased expression specifically in myoepithelial cells (Fig. 11).

To test whether Wnt4 was furthermore required for Adamts18 expression, we engrafted contralateral cleared fat pads with $W T$. 
$\mathrm{EGFP}^{+}$and $\mathrm{Wnt4}^{-/-}$.EGFP ${ }^{+}$mammary epithelia and harvested the transplanted glands on day 8.5 of pregnancy when Wnt4dependent canonical Wnt signaling activity peaks ${ }^{6}$. Levels of Wnt4 expression in the mutant grafts were $1 \%$ of $W T$ levels and Adamts18 expression was reduced to $35 \%$ of $W T$ levels (Fig. $1 \mathrm{~m}$ ). Thus, increased canonical Wnt signaling induces Adamts18 expression and both $P R$ and Wnt4 are required for Adamts 18 mRNA expression. This indicates that myoepithelial Adamts18 expression is downstream of the luminal PR/Wnt4 axis.

Potentially, our conclusion could be confounded by lineage differentiation and cell specification defects resulting from $P R$ and Wnt4 deletions. In light of the finding that both $P R^{-/}$and $W n t 4^{-1-}$ epithelial cells can differentiate into milk secreting alveolar cells ${ }^{29,30}$, major cell specification defects are improbable. Nevertheless, we examined the possibility of a lineage differentiation defect by determining the ratio of luminal and myoepithelial cells in the two mutants. FACS analysis of lineage-depleted WT and $P R^{-1-}$ mammary cells showed no significant difference in the two cell lineages (Supplementary Fig. 1a). As the Wnt4-1- mice die on embryonic day 13, we resorted to transplanting WT.EGFP ${ }^{+}$ and $W n t 4^{-1-}$.EGFP ${ }^{+}$mammary epithelia derived for embryonic mammary buds ${ }^{6}$ to contralateral fat pads and quantified the percentage of Sma+ epithelial cells by IF. The percentage of myoepithelial cells was decreased from $34 \%$ in the $W T$ to $26 \%$ in the $W_{n t 4^{-/}}$epithelium (Supplementary Fig. 1b). To gain more insights into the lineage deregulation, we went on to compare FACS-sorted GFP+ luminal and myoepithelial cells from conditionally Wnt4-deleted (MMTV::Cre ${ }^{+}$.Wnt4l/fl.mT/ $m G)$ and control (MMTV::Cre $e^{+}$.Wnt $\left.4^{w t / w t} . m T / m G\right)$ epithelia by Affymetrix microarray analysis. The number of genes differentially expressed between the two genotypes was almost twice as high in the myoepithelial than in the luminal cell populations (Supplementary Fig. 1c-e). Hence, despite a lineage defect, there are major gene expression changes in the myoepithelium. Gene set enrichment analysis (GSEA) of the differentially expressed genes revealed that signatures reflecting the activity of the canonical Wnt signaling target, $M y c$, and the expression of its target genes were decreased in the $W n t 4^{-/-}$myoepithelial but not luminal cells (Supplementary Fig. 1f). Together these findings are consistent with the model that Wnt 4 secreted by luminal cells activates canonical Wnt signaling in the myoepithelial cells ${ }^{6}$. Wnt4 was the most significantly down-modulated gene in the luminal compartment (Supplementary Fig. 1d). While expression of Cytokeratin $5(\mathrm{Krt5})$ a gene typically enriched in myoepithelial cells, was increased in the Wnt4-/- luminal cells no cell typerelated gene signatures were identified (Supplementary Fig. 1d). In the myoepithelial cell population, the secreted Wnt signaling inhibitor, Wif1, was the most significantly down-modulated gene suggesting the existence of a negative feedback loop in intraepithelial homeostasis (Supplementary Fig. 1e). The stem and progenitor cell markers, Sox 9 and Lgr5, were decreased (Supplementary Fig. 1e). Adamts18 was also among the down modulated genes but failed to reach statistical significance (Supplementary Fig. 1e). GSEA revealed furthermore a decreased stem cell signature and an increase in Tgf- $\beta$ targets in the $W_{n t 4^{-1}}$ myoepithelial cells (Supplementary Fig. 1g). Reactome pathway analysis revealed a protein interactome centered around cell-cell junction and cell junction organization as well as cell-cell communication (Supplementary Fig. 1h). Taken together, while the deletion of Wnt4 results in a stem cell defect with some consequent cell lineage defect, the gene is expressed in the luminal compartment and its deletion affects transcription mostly in the myoepithelial compartment where Adamts18 is expressed.
Mammary gland development in Adamts18 ${ }^{-/-}$mice. To assess the functional importance of Adamts18 in mammary gland development, we generated mice homozygous for an allele lacking exons 8 and 9, which encode the Zn-binding catalytic site ${ }^{31}$ and analyzed their inguinal mammary glands at critical developmental stages by whole mount stereomicroscopy. In prepubertal, 14-dayold $W T$ and Adamts $18^{-/}$littermates, the ductal system was rudimentary and of similar size in both genotypes (Fig. 2a). Consistently, extent of fat pad filling (Fig. 2b) and the number of branching points were comparable in prepubertal, 14-day-old, WT and Adamts $18^{-/-}$littermates (Fig. 2c). In pubertal, 4-6-week-old, WT females, milk ducts grew by characteristic dichotomous branching, extended beyond the subiliac lymph node, and had enlarged tips, terminal end buds (TEBs) characteristic of this stage (Fig. 2d). In the Adamts18 $18^{-/}$littermates, ducts barely reached the lymph node (Fig. 2d). The extent of fat pad filling was reduced by $50 \%$ (Fig. 2e), the number of branching points by $60 \%$ (Fig. $2 \mathrm{f}$ ) and the number of TEBs by $40 \%$ compared to the WT counterparts (Fig. 2g). In adult, 14-week-old, females, the milk ducts reached the edges of fat pads in both genotypes. In $W T$ females, ductal complexity was increased through side branching whereas ducts of the Adamts $18^{-/}$littermates were simple (Fig. $2 \mathrm{~h}$ ) and the number of branching points was $58 \%$ of $W T$ (Fig. 2i). Thus, Adamts 18 is required for ductal development both during puberty and adulthood.

Histological examination of mammary glands from 6-week-old mice revealed structurally normal ducts with intact luminal and myoepithelial layers in both genotypes (Fig. 2j). To address whether the observed delay in ductal elongation was due to increased cell death and/or decreased cell proliferation, we stained sections from pubertal glands for cleaved-caspase 3 and phosphorylated histone $\mathrm{H} 3(\mathrm{pHH} 3)$. The proportion of cleaved caspase3+ cells did not differ significantly (Fig. 2k) but the pHH3-index in Adamts $18^{-/}$mammary epithelia was reduced to $64 \%$ of $W T$ levels (Fig. 2l, m). Thus, the delayed ductal elongation is due to decreased cell proliferation.

Adamts 18 function in the mammary epithelium. Adamts $18^{-/-}$ pups show a transient growth delay ${ }^{18}$, which may indirectly affect mammary gland development. In addition, subfertility associated with abnormalities in the female reproductive tract, such as dorsoventral vagina or imperforate vagina of Adamts $18^{-/-}$females ${ }^{18}$ precluded analysis of mammary gland development during pregnancy. To discern the epithelial-intrinsic role of Adamts18 in ductal growth at later developmental stages, we grafted mammary epithelium from $W T . E G F P^{+}$and Adamts $18^{-/-}$.EGFP ${ }^{+}$females to contralateral inguinal glands of 3-week-old WT female mice surgically divested of their endogenous epithelium. To unequivocally distinguish the engrafted epithelium from host epithelium that could have been inadvertently left behind during surgery, the donor cells constitutively expressed an enhanced green fluorescent protein $(E G F P)$ under control of a chicken $\beta$-actin promoter ${ }^{32}$. Six weeks after engraftment, outgrowths derived from WT donors filled the host fat pads whereas the contralateral Adamts $18^{-/-}$ epithelia failed to do so (Fig. 3a) and the branching points were decreased by $33 \%$ (Fig. 3b). Twelve weeks after engraftment, both WT.EGFP ${ }^{+}$and Adamts $18^{-/-}$.EGFP ${ }^{+}$outgrowths filled the host fat pads but side branching was decreased in Adamts $18^{-/-}$.EGFP ${ }^{+}$ epithelial grafts (Fig. 3c). Flow cytometry of dissociated glands showed a $30 \%$ reduction in $\mathrm{EGFP}^{+}$cells (Fig. 3d) consistent with decreased cell proliferation resulting in lower epithelial cell numbers and delayed branching. Thus, the mammary branching phenotype in Adamts $18^{-/-}$females is intrinsic to the mammary epithelium. 

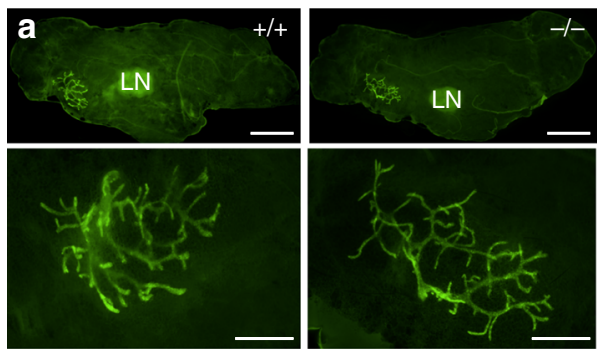

b
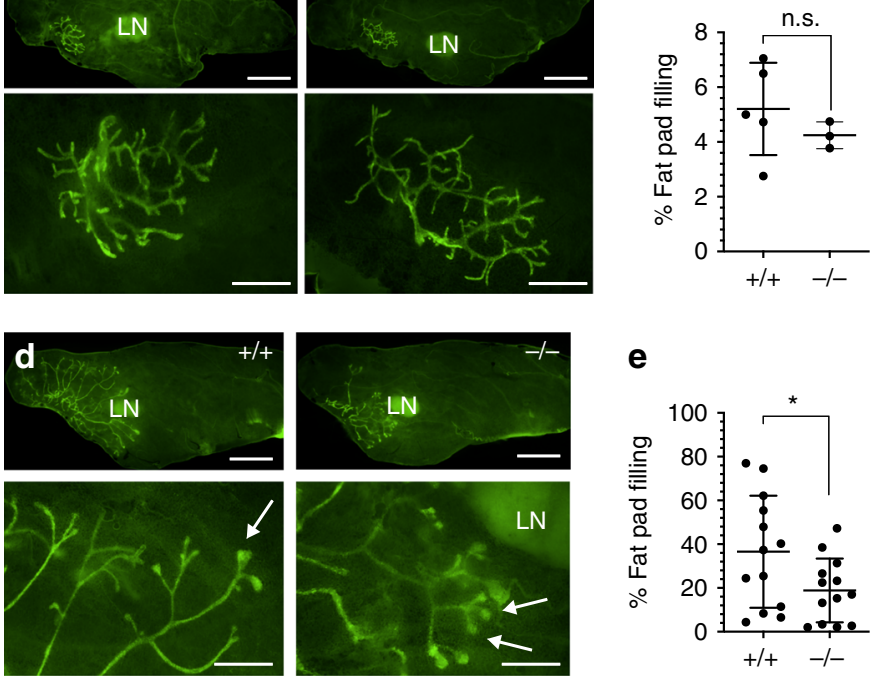

N

h
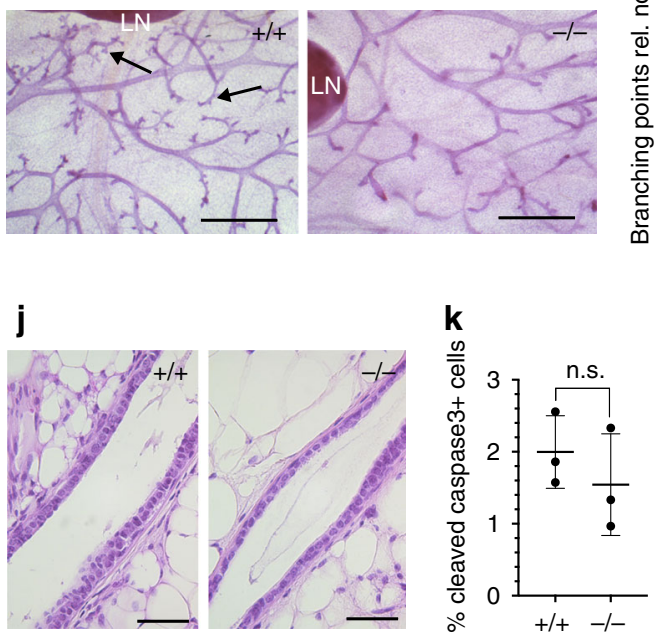

e
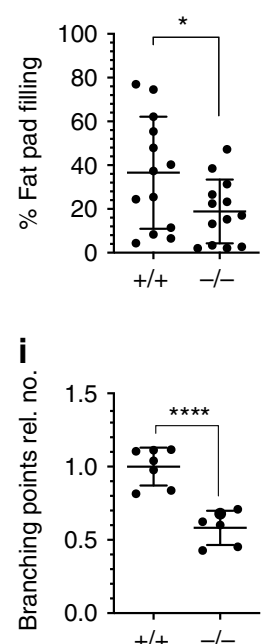

I

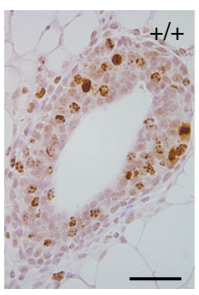

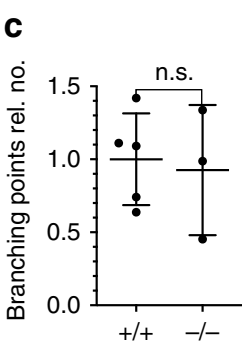

f

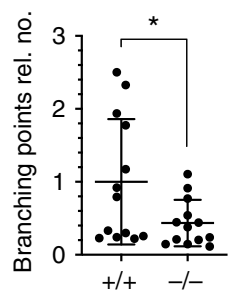

g

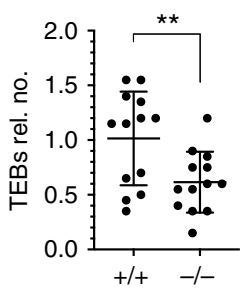

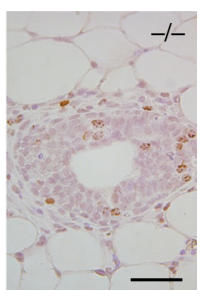

m

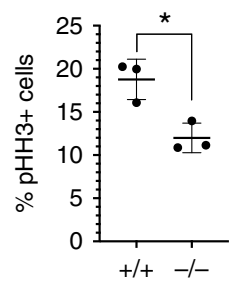

Fig. 2 Mammary gland development in Adamts18 ${ }^{-/-}$mice. a Representative fluorescent stereo-micrographs of inguinal glands from 14-day-old prepubertal WT.EGFP+ and Adamts18 $18^{-/}$.EGFP females; $n=5$ and $n=3$. Scale bar, $500 \mu \mathrm{m}$. b, c Dot plots indicating the percentage of fat pad filling and fold change of branching points on day 14 mammary glands from 5 WT.EGFP+ and 3 Adamts $18^{-/-}$.EGFP vs mice. Data represent mean \pm SD unpaired Student $t$-test, two-tailed. d Representative fluorescent stereo-micrographs of inguinal glands from 4-week-old pubertal WT.EGFP+ and Adamts18- ${ }^{-}$. EGFP mice; $n=13$ for each genotype. LN: subiliac lymph node. Arrows indicate TEBs; scale bar, $500 \mu \mathrm{m}$. e-g Dot plots indicating percentage of fat pad filling, relative number of branching points and TEBs quantified at 4-6 weeks of age. Data represent mean \pm SD from 13 WT.EGFP+ and 13 Adamts18 ${ }^{-/-}$. EGFP mice. Unpaired Student $t$-test, two-tailed. h Representative stereo micrographs of whole mounted inguinal glands from 7 WT and 7 Adamts $18^{-/-} 14-$ weekold virgin mice. Arrows point to side branches; scale bars, $500 \mu \mathrm{m}$. i Dot plot showing relative number of branching points. Data represent mean \pm SD from 7 WT and 7 Adamts $18^{-/-}$14-week-old virgin mice. Unpaired Student $t$-test, two-tailed. j Representative micrographs of H\&E-stained histological sections of mammary glands from 5 -week-old $W T$ and Adamts $18^{-/-}$littermates, $n=4$; scale bar, $50 \mu \mathrm{m}$. $\mathbf{k}$ Dot plot showing percentage of cleaved caspase $3+$ cells in TEBs of WT and Adamts18 ${ }^{-/}$- females. Data represent mean \pm SD from 3 WT and 3 Adamts $18^{-/-}$mice. Unpaired Student $t$-test, two-tailed. I Representative pHH3 (brown) IHC on mammary glands from 6-week-old WT and Adamts $18^{-/-}$females; hematoxylin counterstain; scale bar, $50 \mu \mathrm{m}$. $\mathbf{m}$ Dot plot showing the percentage of $\mathrm{pHH} 3+$ positive cells in TEBs of 3 WT and 3 Adamts18 $-/-$ females. Data represent mean \pm SD, unpaired Student $t$-test, two-tailed. ${ }^{\star} p<0.05 ;{ }^{\star \star} p<0.01 ;{ }^{\star \star \star} p<0.001 ;{ }^{\star \star \star \star} p<0.0001$, n.s. not significant.

At 14.5 days of pregnancy, epithelia of both genotypes showed widespread alveoli both by fluorescence stereomicroscopy and histology (Fig. 3e). At day 1 of lactation, alveoli were fully distended (Fig. 3f) suggesting normal lactogenic function. However, at both time points, spaces between EGFP + epithelial structures were larger in Adamts $18^{-1-}$.EGFP ${ }^{+}$grafts than in the WT counterparts consistent with reduced side branching at earlier stages (Fig. $3 \mathrm{e}-\mathrm{g}$ ). In line with the morphologic analysis and the decreased number of MECs, transcript levels of lactogenic differentiation markers such as Lalba, Wap, and CsnA were lower in mutant glands compared to
WT controls but failed to reach statistical significance when normalized to the epithelial marker Krt18 (Fig. 3h). Thus, while epithelial cell numbers are decreased in the absence of Adamts18, the protease is not required for cytodifferentiation.

Adamts18 expression has been reported in the stromal compartment and was confirmed by semi quantitative RT-PCR analysis of $W T$ fat pads engrafted with Adamts $18^{-/}$epithelium showing 25\% of the Adamts18 transcript levels detected in WT recombinants (Supplementary Fig. 2a). To determine the functional importance of this stromal expression, WT.EGFP ${ }^{+}$ 

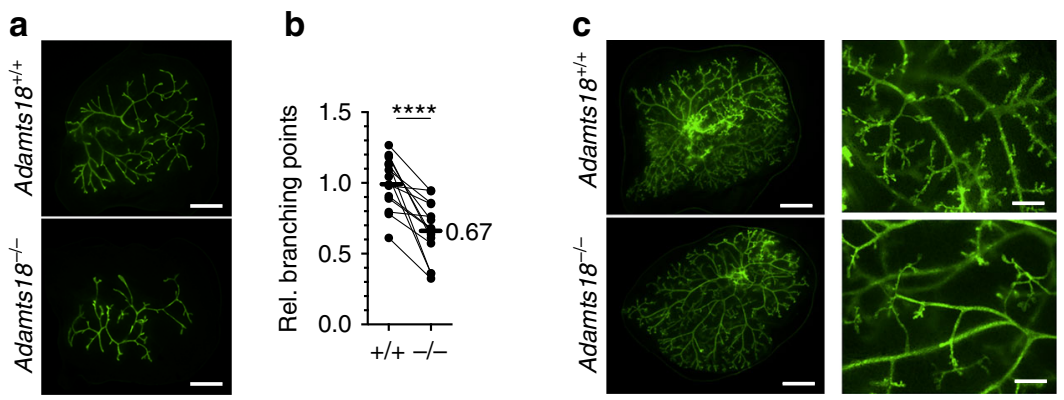

d
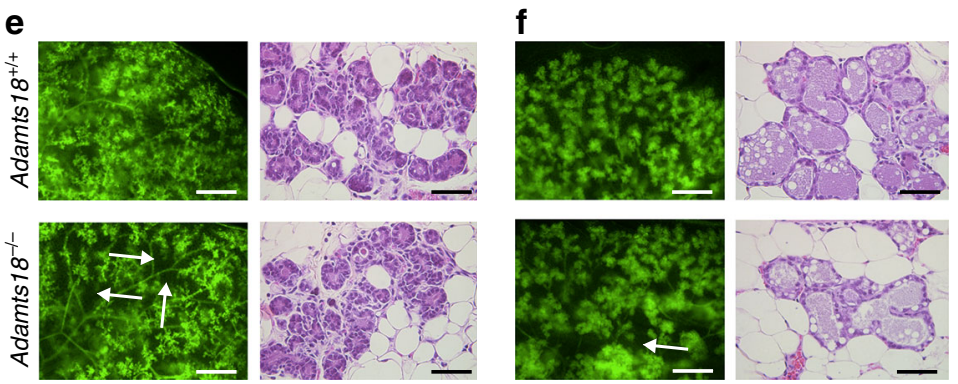

\section{g}

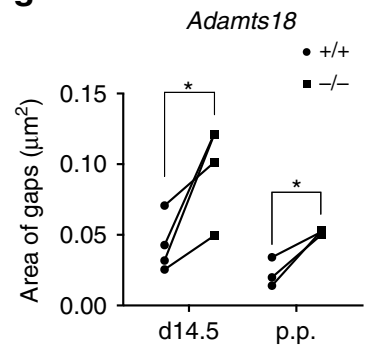

h

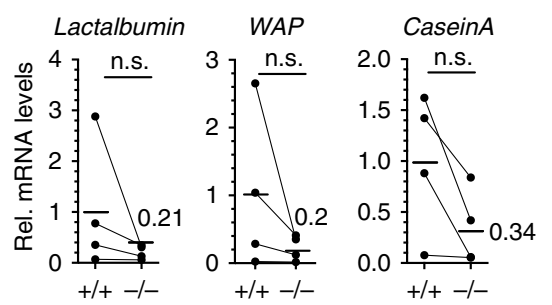

i
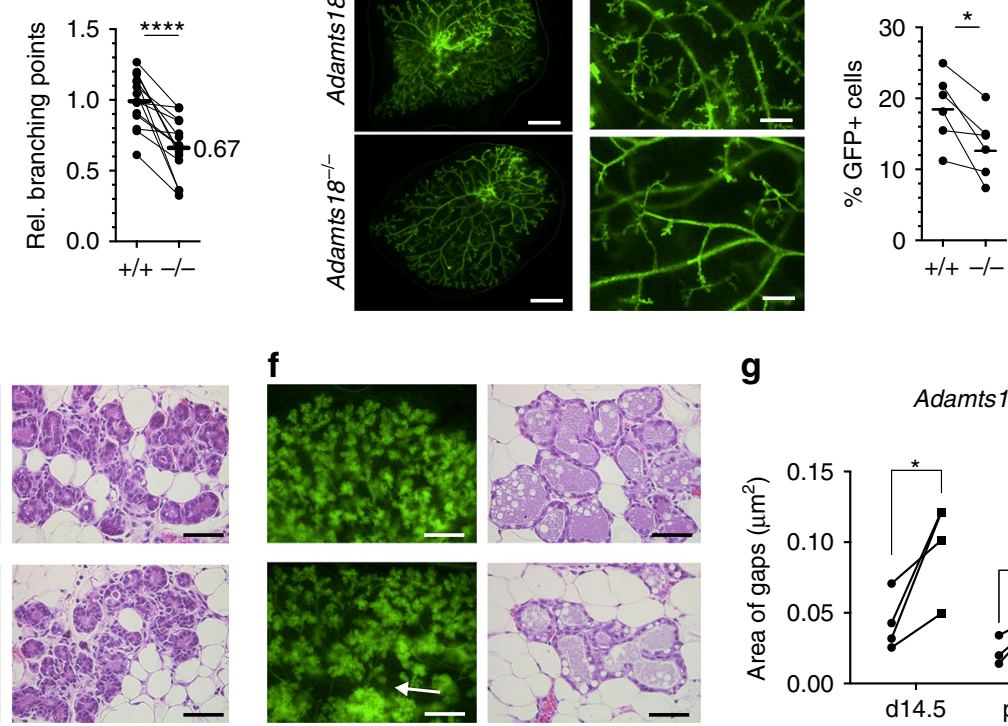

d14.5 p.p.
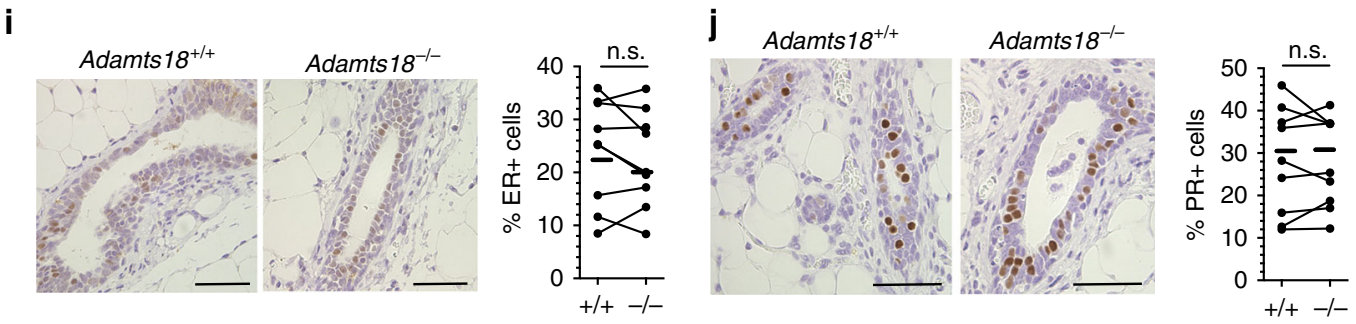

Fig. 3 Mammary epithelial-intrinsic role of Adamts18. a Fluorescence stereo-micrographs of contralateral glands 6 weeks after engraftment with WT.EGFP + or Adamts18 /- EGFP+ epithelia; scale bar, $500 \mu \mathrm{m}$. b Dot plot showing relative number of branching points in 14 contralateral WT.EGFP+ and Adamts $18^{-/-}$. EGFP+ epithelial outgrowths; 4 different donors were used. c Representative fluorescence stereo micrographs of contralateral glands engrafted with WT.EGFP+ or Adamts18-/-.EGFP+ epithelium 12 weeks earlier. Scale bars, $500 \mu \mathrm{m}$ (left), $150 \mu \mathrm{m}$ (right). d Dot plot showing percentage of GFP+ cells obtained from WT. EGFP $^{+}$and Adamts18 $/-$. EGFP+ contralateral grafts by flow cytometry. Tissue from 3 different donors was grafted to 6 pairs of contralateral fat pads.

e Representative fluorescence stereo micrographs and micrographs of H\&E-stained contralateral glands engrafted with WT.EGFP+ (top) and Adamts18 ${ }^{-/}$. EGFP+ (bottom) epithelia from host at day 14.5 of pregnancy. Three different donors were used, $(n=4)$. Arrows show interductal spaces; Scale bars, $5 \mathrm{~mm}$ and $50 \mu \mathrm{m}$. f Representative fluorescence stereo micrographs of 4 pairs of contralateral glands engrafted with WT.EGFP ${ }^{+}$and Adamts $18^{-/-}$.EGFP ${ }^{+}$epithelia from host at lactation, 3 different donors were used in 3 independent experiments. H\&E-stained micrographs thereof; scale bar, $50 \mu \mathrm{m}$. $\mathbf{g}$ Dot plots showing quantification of areas between branches from 4 pairs of contralateral glands at day 14.5 of pregnancy and 3 pairs of contralateral glands at post-partum. $\mathbf{h}$ Dot plots showing relative transcript levels of Lactalbumin, Whey Acidic Protein (WAP), Casein A normalized to Krt18, in contralateral glands transplanted with WT.EGFP ${ }^{+}$and Adamts18-/-.EGFP+ epithelia. Host is 14.5-day pregnant. Each pair of points represents an individual mouse; $n=4$. i Representative micrographs showing IHC for ER on contralaterally engrafted WT.EGFP+ and Adamts18-/-.EGFP+ epithelia. Scale bar, $100 \mu \mathrm{m}$. Dot plot showing percentage of ER+ luminal cells in 9 contralateral grafts. j PR staining on contralateral glands engrafted with WT.EGFP+ and Adamts18 ${ }^{-1-}$.EGFP + epithelia. Scale bar, $50 \mu \mathrm{m}$. Dot plot showing percentage of PR+ luminal cells in 10 contralateral grafts. Statistical analysis by paired Student $t$-test, two-tailed. ${ }^{\star} p<0.05 ;{ }^{\star \star} p<0.01 ;{ }^{* \star \star} p<0.001 ;{ }^{\star \star \star \star} p<$ 0.0001 , n.s. not significant.

mammary epithelium was transplanted into cleared inguinal mammary fat pads of 3-week-old Adamts $18^{-/-}$and WT mice. The recombined tissues were contralaterally transplanted unto the abdominal muscles of adult WT females. Fluorescence stereomicroscopy 6 weeks later showed that $W T$ donor epithelium filled both $W T$ and Adamts $18^{-/-}$fat pads to comparable extent (Supplementary Fig. 2b, c). Twelve weeks after surgery, in both
WT and Adamts18 $1-$ fat pads the implanted epithelia had developed side branches to comparable extent (Supplementary Fig. 2d). Thus, stromal Adamts18 expression is not required for ductal branching.

As epithelial ER and PR signaling drive pubertal dichotomous branching and estrous cycle-induced side branching, respectively ${ }^{29,33}$, we asked whether receptor expression was affected by 
Adamts18 inactivation. IHC of sections from contralateral glands engrafted with WT.EGFP ${ }^{+}$and Adamts $18^{-/-} . E G F P^{+}$epithelia revealed comparable proportions of ER + (Fig. 3i) and PR + cells (Fig. 3j) indicating that Adamts18 is not required for ER or PR protein expression.

The role of Adamts18 in mammary epithelial self-renewal. Delayed pubertal ductal outgrowth and reduced side branching together with normal alveologenesis and cytodifferentiation were previously observed in $W n t 4^{-1-}$ epithelia ${ }^{26}$ and shown to result from a stem cell defect ${ }^{6}$. To test whether Adamts 18 deletion also affects mammary stem cells (MaSCs), we analyzed cells from dissociated $W T$ and Adamts $18^{-1-}$ mammary glands by $\mathrm{FACS}^{34}$ using CD24 and CD49f detection after depletion for lineage positive cells (Fig. 4a). The number of lineage-depleted cells obtained from mammary glands of 14-week-old females was one third less in Adamts $18^{-/-}$compared to WT (Fig. 4b). The percentage of both luminal ( $\mathrm{Lin}^{-} \mathrm{CD} 24^{+} \mathrm{CD}^{-} 9 \mathrm{f}^{-}$) and myoepithelial ( $\mathrm{Lin}^{-} \mathrm{CD} 24^{\text {low }} \mathrm{CD} 49 \mathrm{f}^{\text {low }}$ ) cells was not significantly altered in Adamts $18^{-/-}$glands but the stromal cell fraction (Lin ${ }^{-}$ CD24- ${ }^{-}$CD $49 \mathrm{f}^{-}$) increased by $19 \%$ in the mutant glands (Fig. 4c). Mammary progenitors, which give rise to colonies and are called colony forming cells (CFCs) represented $<1 \%$ of the lineage negative cells in both genotypes whereas the number of MaSCs (Lin ${ }^{-} \mathrm{CD} 24^{\text {med }} \mathrm{CD} 49 \mathrm{f}^{\text {high }}$ ) also defined as mammary repopulating units (MRUs) was decreased by $43 \%$ in Adamts $18^{-/-}$glands (Fig. 4a, d).

To functionally evaluate stem cell frequency in $W T$ and Adamts $18^{-/-}$mammary epithelia, we injected serially diluted single cells from WT.EGFP ${ }^{+}$and Adamts $18^{-/-}$.EGFP ${ }^{+}$mammary glands to contralateral cleared fat pads of 3-week-old WT.EGFPfemale mice. After 8 weeks, we determined frequency and extent of outgrowth by fluorescence stereomicroscopy combined with image analysis. The repopulating cell frequency ${ }^{35}$ of Adamts $18^{-/-}$ cells was $10 \%$ of the $W T$ cells with $1 / 20,000$ vs. $1 / 2000$ (Fig. 4 e).

The single cell-based in vivo reconstitution assay can be confounded by cell adhesion and/or cell migration defects as well as by increased susceptibility to apoptosis. All these factors impact on any cell's ability, whether stem cell or not, to establish itself after injection in the fat pad, which is, of course, a prerequisite for the generation of any progeny. A complex assay that overcomes these limitations is the serial transplantation of pieces of intact epithelium. WT epithelium that is serially grafted can fill cleared fat pads for up to 7 generations ${ }^{36}$. Indeed, WT. $E G F P^{+}$epithelium filled host fat pads efficiently over 5 transplant generations, however, the reconstitution ability of the contralaterally grafted Adamts $18^{-/-}$.EGFP ${ }^{+}$epithelium decreased progressively to cease completely upon the 5 th transplant (Fig. $4 \mathrm{f}, \mathrm{h}$, i). Histological analysis of the 4th generation transplants by $\mathrm{H} \& \mathrm{E}$ revealed no obvious difference (Fig. 4g). Thus, Adamts18 is required for the regeneration capacity of the mammary epithelium, albeit to a lesser extent than $P R$ and $W n t 4$, whose deletion blocks reconstitution at the 4 th and 3 rd generation, respectively, by the same assay ${ }^{6}$.

The basement membrane is part of the stem cell niche. To address the mechanisms by which Adamts 18 affects stem cell activity, we searched for its binding partners. In light of the myoepithelial cell-specific expression of the protease, we chose the human breast epithelial cell line, MCF10A, which has myoepithelial/basal characteristics ${ }^{37}$, as a model. We ectopically expressed V5-tagged ADAMTS18 in these cells, immune precipitated it from the conditioned medium, and analyzed coimmunoprecipitated proteins by mass spectrometry. We discovered 238 proteins cumulatively in 3 independent experiments
(Supplementary Data 1), of which 31 were identified in $\geq 2$ experiments (Fig. 5a). Transforming Growth Factor Beta-Induced (TGFBI), a secreted molecule that contains RGD domains similar to fibronectin and laminin and inhibits cellular adhesion to the ECM, was among the 12 proteins identified in all 3 experiments $^{38}$. Bioinformatic analysis with MetaCore showed that top enriched MetaCore processes related to ECM organization and hemidesmosome assembly (Fig. 5b, Supplementary Table 1). The top localizations of the putative ADAMTS18 interactors were ECM, laminin-5 complex, and BM (Fig. 5c, Supplementary Table 2). Together, these findings support the hypothesis that Adamts18 function relates to the ECM and, more specifically, to the connection between epithelium and BM. This implies that the BM may be part of the stem cell niche.

To seek in vivo evidence for a role of the BM as part of the stem cell niche we turned to mice deficient for Col18a1 because this heparin-sulfate proteoglycan is specifically localized to $\mathrm{BMs}^{39}$. Whole mount stereo-microscopy and morphometric analysis showed that Col18a1 ${ }^{-/}$females like their Adamts18 ${ }^{-/}$counterparts had delayed ductal elongation and fewer TEBs compared to their WT littermates (Fig. 5d). Adamts18 and Col18a1 doubledeficient $(D K O)$ mice showed a further decrease in TEB numbers, fat pad filling, and branching points at 6 weeks compared to single knockouts (Fig. 5e) indicating that Adamts18 and Col18a1 have additive roles in ductal elongation. To assess whether this genetic interaction affects stem cell function, we serially transplanted the $D K O$ epithelium. While the contralateral WT epithelium reconstituted glands over 5 transplant cycles, the DKO epithelium failed to reconstitute by the $3 \mathrm{rd}$ generation (Fig. $5 \mathrm{f}-\mathrm{h}$ ). Thus, Adamts 18 and Col18al cooperate in mammary stem cell control, providing in vivo evidence for a role of the BM in stem cell function, likely as part of the stem cell niche.

Adamts18 modulates the ECM. To probe for structural alterations in the ECM related to Adamts18 deletion, we used picrosirius red to stain Adamts $18^{-/-}$and WT pubertal mammary glands. Fibrillar collagen was increased around the ducts and TEBs in Adamts $18^{-/-}$relative to $W T$ (Fig. 6a). Immunoblotting of protein lysates from pubertal WT and Adamts $18^{-/-}$glands and quantification showed that levels of the important BM components, laminin and collagen IV increased 1.7- and 3.9-fold, respectively, in Adamts18 ${ }^{-/-}$glands (Fig. 6b, c). Levels of the major fibrillar collagen, collagen I, were increased 6.2-fold (Fig. 6b, c). Assembly of nascent collagen I, laminin and collagen IV matrices rely on initial assembly of fibrils composed of the primordial ECM glycoprotein fibronectin, the first ECM protein to be expressed during tissue development and wound healing ${ }^{40,41}$. Fibronectin levels were 3.2fold higher in the mutants than in WT (Fig. 6b, c). IF showed increased staining intensity for all these proteins around ducts and TEBs in Adamts18 ${ }^{-/}$relative to WT pubertal glands (Fig. 6d). The staining was restricted to the BM for laminin and collagen IV but extended to the interstitial ECM for collagen I and fibronectin. Thus, in the absence of Adamts18, major ECM/BM components accumulate in the pubertal mammary gland in line with an important role for Adamts18 in ECM/BM remodeling.

Interestingly, analysis of mammary glands from 14-week-old WT and Adamts $18^{-/}$littermates showed that protein levels of laminin, collagens I and IV as well as fibronectin did not differ significantly between the two genotypes (Fig. 6e, f). This shows that Adamts18 is critical for ECM/BM modulation during pubertal ductal elongation and suggest that this specific developmental window determines mammary stem cell function.

Adamts18 cleaves fibronectin. In contrast with the increased fibronectin protein levels, its mRNA levels were unaltered in the 
a

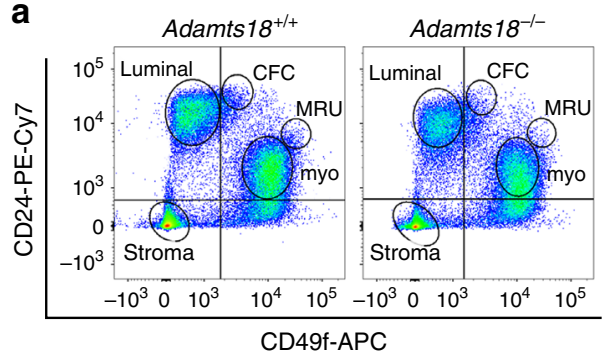

b

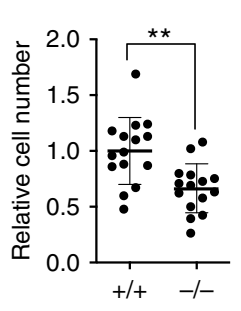

C

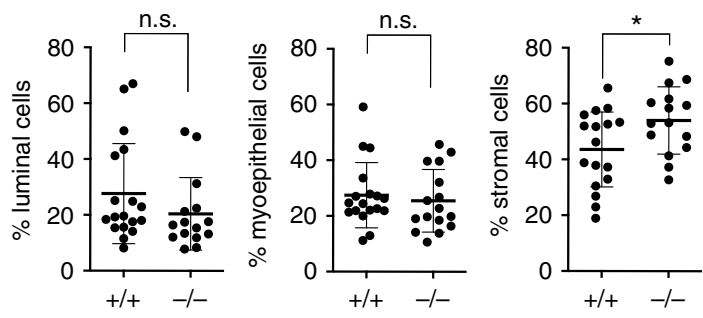

d

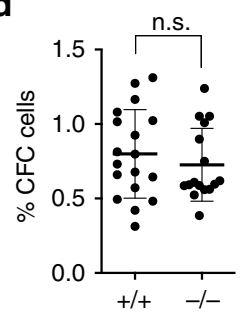

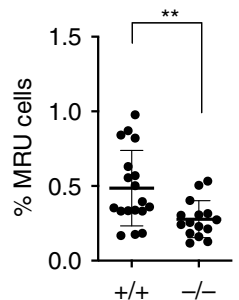

e

\begin{tabular}{|c|c|c|}
\hline \# of cells injected & \multicolumn{2}{|c|}{ \# positive outgrowth / total \# of fat pads engrafted } \\
\hline & Adamts $18^{+/+}$ & Adamts18 \\
\hline 100,000 & $5 / 5$ & $4 / 5$ \\
\hline 10,000 & $4 / 5$ & $3 / 6$ \\
\hline 1000 & $6 / 6$ & $3 / 6$ \\
\hline 100 & $5 / 6$ & $1 / 6$ \\
\hline $\begin{array}{c}\text { Repopulating } \\
\text { frequency }\end{array}$ & $1 / 2071$ & $1 / 20,793$ \\
\hline
\end{tabular}

f
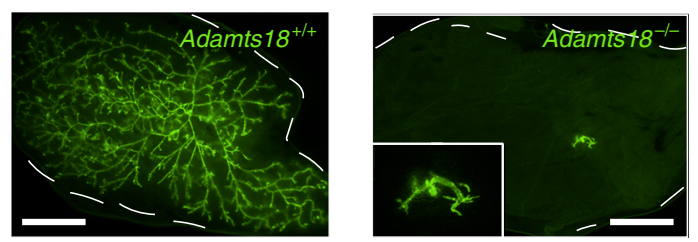

g
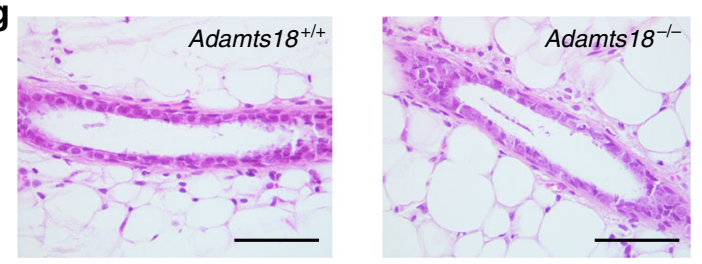

h

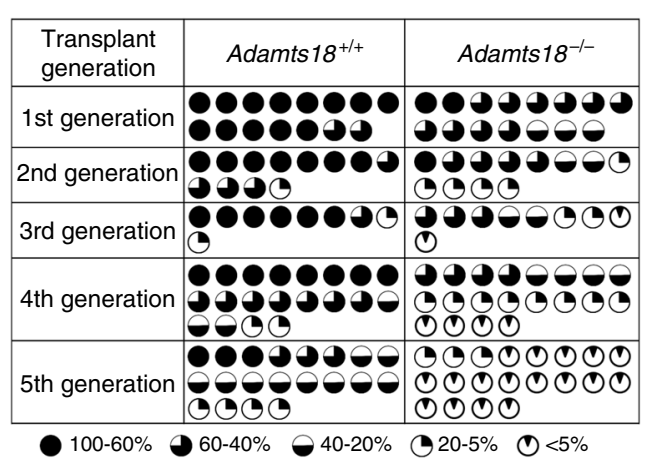

i

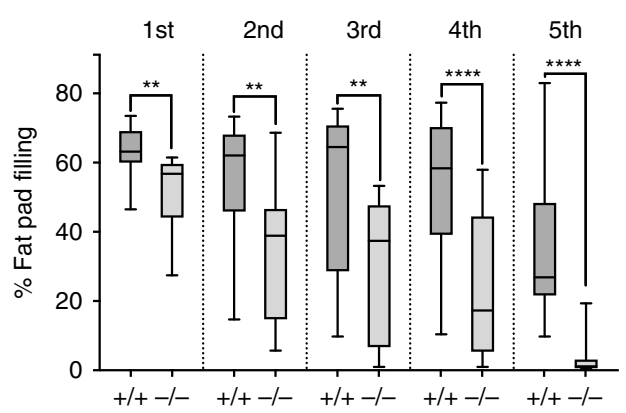

Fig. 4 Role of Adamts18 in the regenerative capacity of the mammary epithelium. a Representative FACS dot plot showing CD49f and CD24 expression in the Lin ${ }^{-}$mammary cells from 14-week-old WT and Adamts18-/- littermates. b Relative number of cells isolated from 15 14-week-old WT and Adamts18-/littermates. c, $\mathbf{d}$ Dot plots showing lineage negative mammary cell populations from the mammary glands of 14-week-old WT and Adamts18-/- littermates. Data represent mean \pm SD from 18 WT and 15 Adamts $18^{-/-}$mice. Unpaired Student $t$-test, two-tailed. Total number of cells (b), percentage of luminal, myoepithelial, stromal cells (c), CFC and MRUs (d). Data represent mean \pm SD from 18 WT and 15 Adamts $18^{-/-}$mice. Unpaired Student $t$-test, two-tailed. e Table showing mammary outgrowths derived from WT.EGFP+ and Adamts18-/-.EGFP+ mammary cells injected at limiting dilutions into cleared contralateral fat pads. Positive outgrowths are defined as $>5 \%$ fat pad area filled and related to total number of transplants. Repopulating cell frequency is shown, data are pooled from 3 independent experiments. f Fluorescence stereo-micrographs of contralateral mammary fat pads of recipient mice at the 5th generation of serial transplant after 8-12 weeks; scale bar, $500 \mu \mathrm{m}$. $\mathbf{g}$ Micrographs of H\&E- stained histological sections of 4th generation transplants; scale bar, $50 \mu \mathrm{m}$. $\mathbf{h}$ Table summarizing 3 independent serial transplant experiments with WT.EGFP+ and Adamts $18^{-/}{ }^{-}$.EGFP ${ }^{+}$epithelia. Each engrafted gland is represented by a micrograph; black sectors represent area of fat pad filled by grafted epithelium. $\mathbf{i}$ Box plot showing extent of fat pad filling of contralateral grafts in each transplant generation. Boxes span the 25 th to 75 th percentile, whiskers 1.5 times the interquartile range. $p$-Values were determined by Wilcoxon test. ${ }^{\star} p<0.05$; ${ }^{\star \star} p<0.01 ;{ }^{\star \star \star} p<0.001 ;{ }^{\star \star \star \star} p<0.0001$, n.s. not significant.

pubertal Adamts18 $18^{-/}$mammary glands (Fig. 6g) suggesting that the observed increased staining could result from translational or posttranslational changes attributable to lack of Adamts18. As fibronectin is the prime component of nascent ECM fibers and a substrate of the Adamts18 homolog Adamts16, we tested whether it is equally an Adamts18 substrate. We purified the secreted active form of ADAMTS18 from HEK-293T cells and incubated it with $\mathrm{N}$-terminal $70 \mathrm{kDa}$ fibronectin. The exogenous fibronectin fragment migrated slightly faster when co-incubated with EDTA and was undetectable in the presence of ADAMTS18 after $24 \mathrm{~h}$. When the digest was supplemented with EDTA, which chelates the bivalent metal ions required for ADAMTS activity, no change in fibronectin abundance was seen (Fig. 6h). Additionally, HEK293 T cells expressing ADAMTS18 or a control vector were incubated without or with the $70 \mathrm{kDa}$ recombinant fibronectin. By western blot, the medium of cells expressing ADAMTS18, but not the control vector, showed a readily detectable $30 \mathrm{kDa}$ fibronectin fragment (Fig. 6i) similar to that detected after 
a

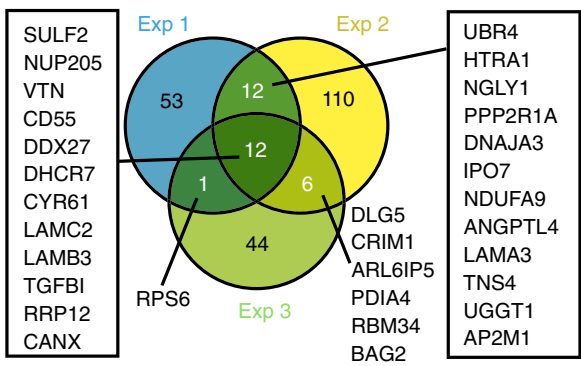

b

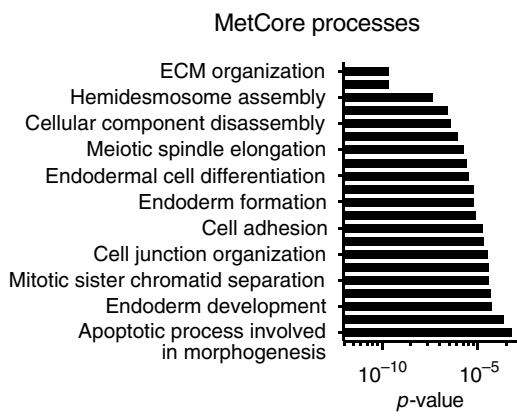

c

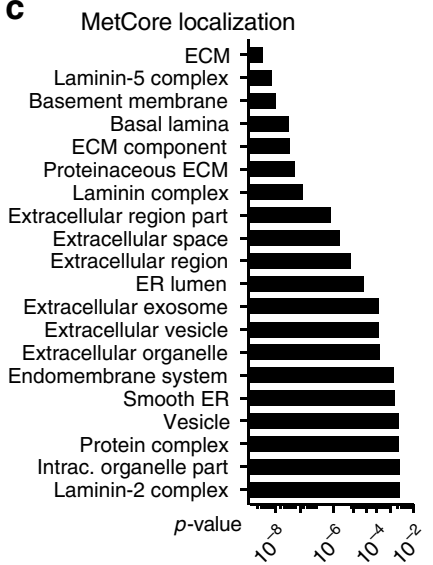

d
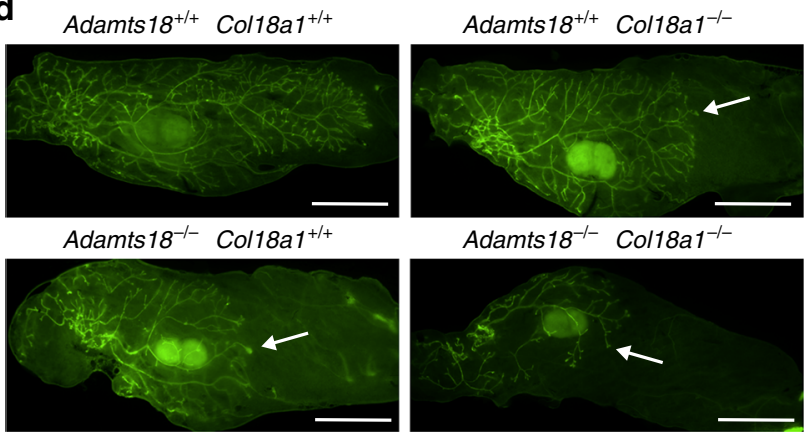

e

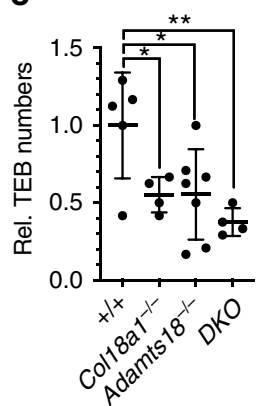

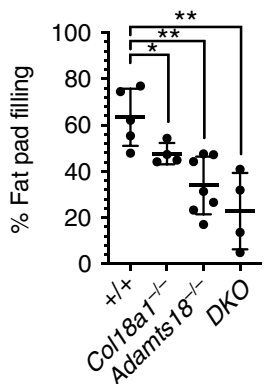

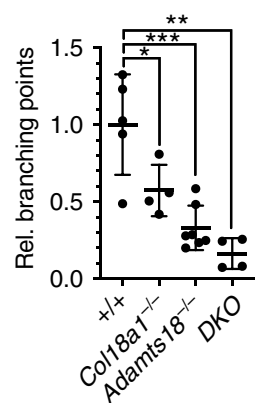

f
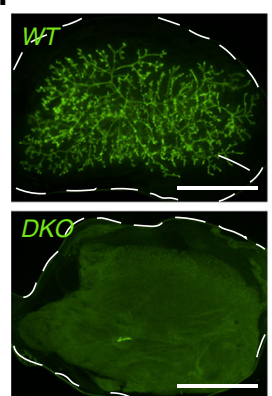

g

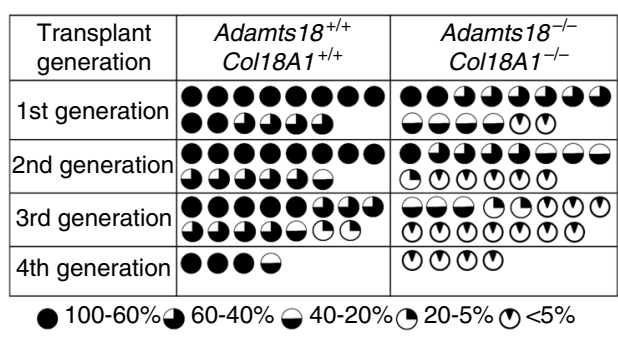

h Transplant generation

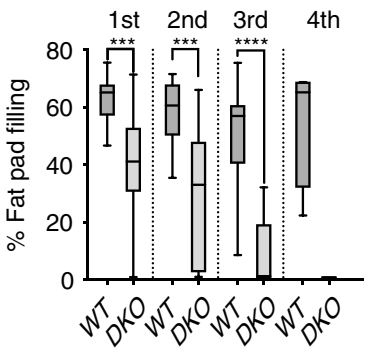

Fig. 5 BM proteins and glycoproteins are involved in stem cell function. a Venn diagram showing candidate ADAMTS18 binding proteins in the supernatant of MCF10A cells identified by affinity purification mass spectrometry. Gene names are shown in boxes for ease of recognition. Three independent experiments were done at different times. $\mathbf{b}$ Bar graph showing the top 20 METACORE processes of candidate ADAMTS18 binding partners based on $p$-values. $\mathbf{c}$ Bar graph showing top 20 METACORE localizations of candidate ADAMTS18 binding partners based on $p$-values. $\mathbf{d}$ Fluorescent stereo micrographs of representative 4th gland from WT.EGFP+ ${ }^{+}$Col18a1 ${ }^{-/-}$.EGFP+ ${ }^{+}$, Adamts18-/-.EGFP ${ }^{+}$and double-deficient (DKO).EGFP+ 6-week-old pubertal mice; $n=5,4,7$ and 4. Arrows indicate TEBs, scale bar, $500 \mu \mathrm{m}$. e Dot plots indicating the relative TEB numbers, percentage of fat pad filling, and relative branching points at 6 weeks of age. Data represent mean \pm SD from 5 WT, 4 Col18a1-/-, 7 Adamts $18^{-/-}$, and 4 DKO mice. One-way analysis of variance (ANOVA). $\mathbf{f}$ Fluorescence stereo-micrographs of contralateral mammary fat pads of Rag1-/- recipient mice with 4 th-generation mammary outgrowths derived from WT.EGFP+ and DKO.EGFP+ donor mice after 8-12 weeks; scale bar, $500 \mu \mathrm{m}$ and H\&E-stained sections thereof, scale bar $100 \mu \mathrm{m}$. $\mathbf{g}$ Table summarizing 3 independent serial transplant experiments with WT.EGFP ${ }^{+}$and DKO.EGFP+ epithelia. Each engrafted gland is represented by a micrograph; black sectors represent the area of the fat pad filled by engrafted epithelium. $\mathbf{h}$ Box plot showing extent of fat pad filling of WT.EGFP+ and DKO.EGFP+ contralateral grafts in each transplant generation. Boxes span the 25th to 75 th percentile, whiskers 1.5 times the interquartile range. $p$-Values were determined by Wilcoxon test. ${ }^{\star} p<0.05 ;{ }^{\star \star} p<0.01$; ${ }^{\star \star \star} p<0.001 ;{ }^{\star \star \star \star} p<0.0001$, n.s. not significant.

cleavage by Adamts $16^{21}$. The amount of cleaved fibronectin increased 16-fold in the presence of ADAMTS18 (Fig. 6j). Thus, the presence of ADAMTS18 leads to fibronectin proteolysis, which may influence abundance of other ECM proteins and indirectly regulate growth factor availability and signaling.

Stem cell signaling in Adamts18 ${ }^{-/-}$glands. Our findings pointed to the observed stem cell defect being secondary to changes in the ECM/BM. To elucidate the mechanisms by which altered ECM affected stem cell signaling, we transcriptionally profiled 3 pairs of contralateral glands engrafted with either $W T$. $E_{G F P}+$ or Adamts $18^{-/}$. EGFP ${ }^{+}$epithelia using RNA-seq. PCA analysis was used to identify and visualize possible batch effects due to sources of variation in the mice used (Supplementary Fig. 3a). After removing these effects by applying 2-way ANOVA correction, samples clustered by biological subgroups (Supplementary Fig. 3b). Expression of Adamts18, Fgfr2, and Ctgf was tested and found reduced in all 3 Adamts $18^{-/-}$samples after read 
a
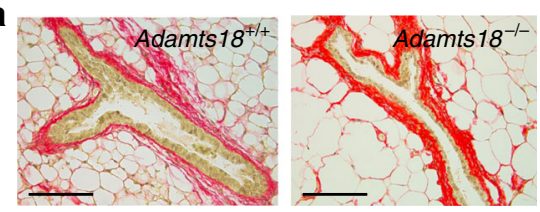

b

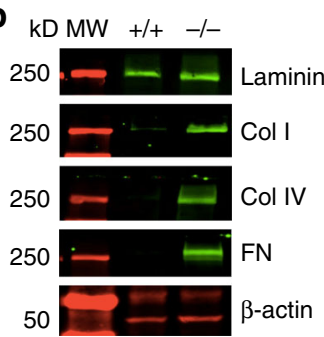

C
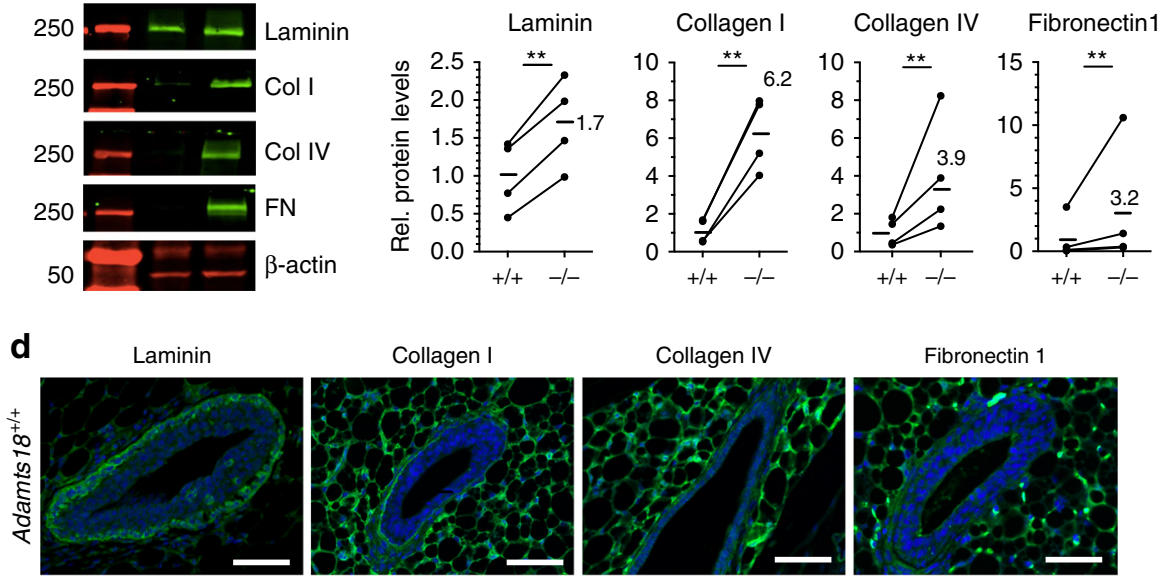

Fibronectin 1
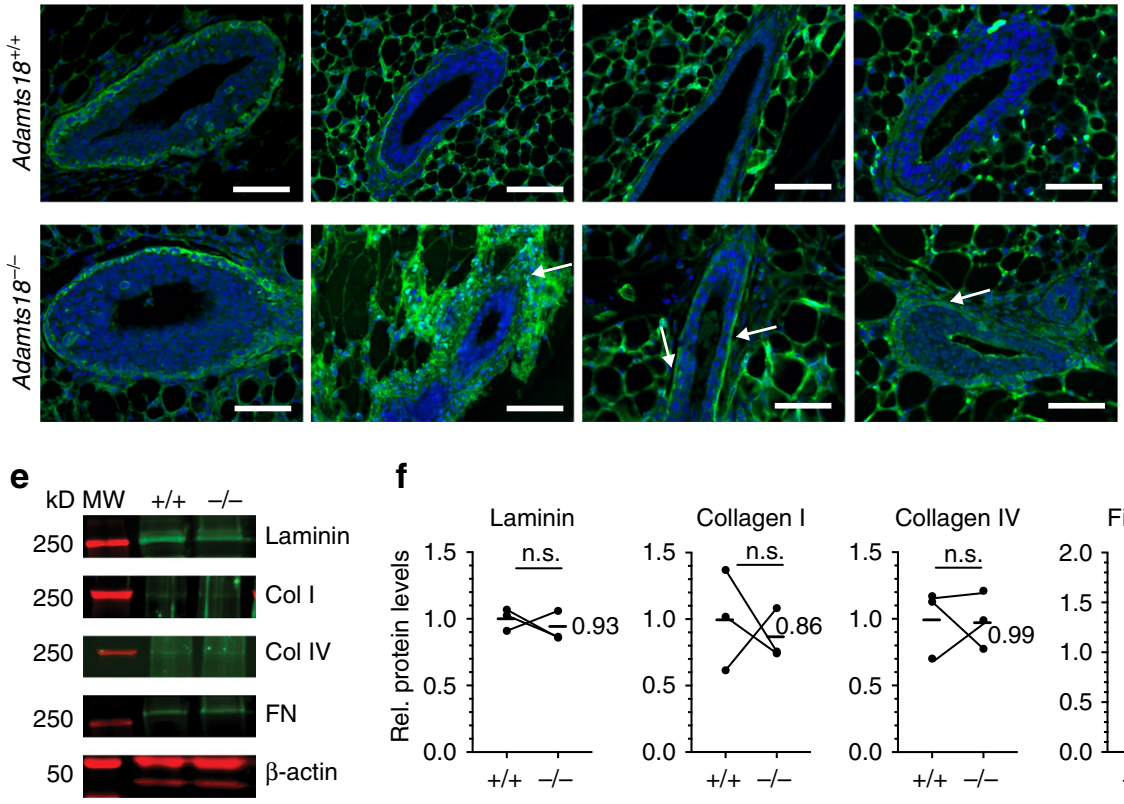

f
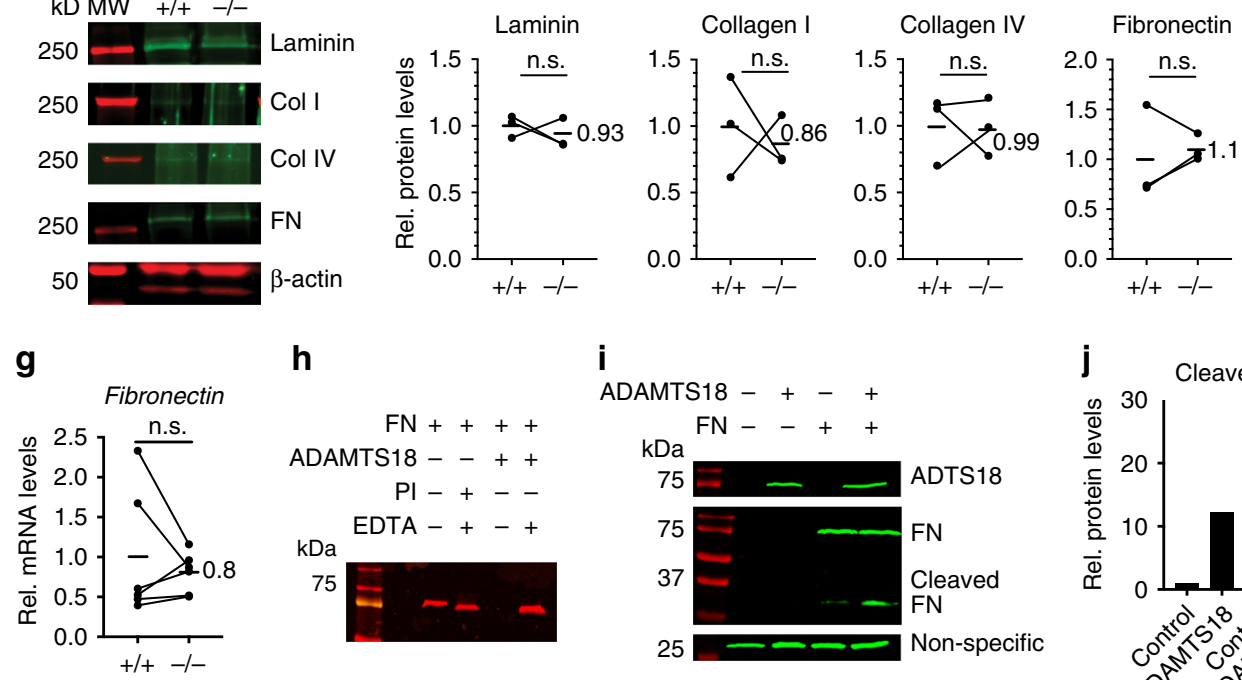

h

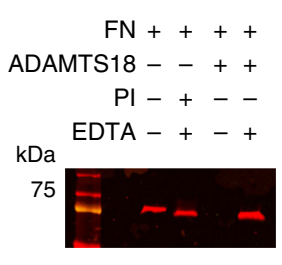

i ADAMTS18 - + - +
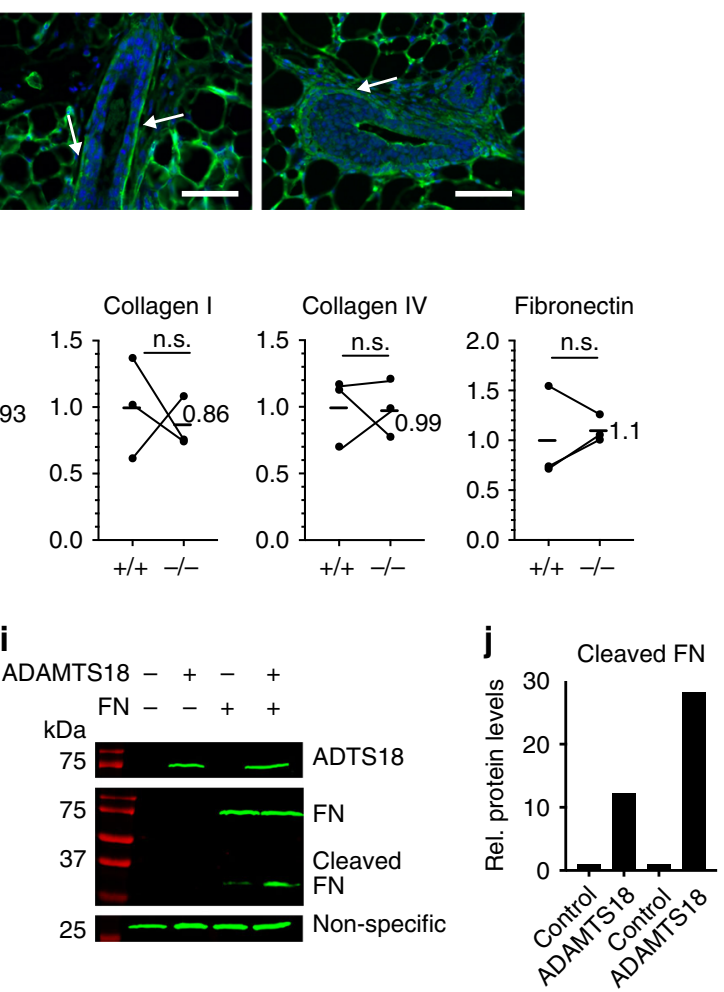

count normalization (Supplementary Fig. 3c). Overall, in Adamts $18^{-/-}$transplanted glands, expression of 313 genes decreased $(\mathrm{FC}<0.8, p<0.05)$ and that of 273 genes increased (FC $>1.25, p<0.05$ ) (Fig. 7a). Analysis of the differentially expressed genes by pathway enrichment analysis using both ReactomePA ${ }^{42}$ and ClusterProfiler ${ }^{43}$ showed that cell junctions and ECM were affected, in particular various collagens and laminins (Supplementary Fig. 3d-g). More specifically, out of 40 significant GO terms, 11 were related to the ECM and 10 to Fgfr signaling, a pathway critical for stem cells ${ }^{44,45}$ (Supplementary Table 3). Two of the 40 terms related to Hippo-Yap/Taz signaling another pathway critical for stem cell differentiation, which is upstream of $F g f 2^{46}$. When we specifically interrogated the genes whose

expression decreased, Reactome pathway analysis revealed Yap/ Taz-mediated gene expression (Fig. $7 \mathrm{~b}$ ) and a protein interactome centered around cell-cell communication and cell-cell junctions as well as ECM, laminin and collagen complexes and assembly (Fig. 7c) that partly overlap with the Wnt4 specific interactome (Supplementary Fig. 1h).

In light of the increased ECM deposition, the differential expression of various ECM-related genes as well as the involvement of the Yap/Taz signaling pathway, we evaluated integrin expression in the Adamts $18^{-/-}$glands. We extracted 27 Integrin genes, $\alpha$ and $\beta$ Integrin subunits, from the RNAseq analysis and generated a heatmap (Supplementary Fig. 3h). No integrin-related gene was significantly altered by adjusted $\mathrm{p}$-value, 
Fig. 6 Biochemical changes and Fibronectin cleavage elicited by Adamts18. a Representative picrosirius red staining for fibrillar collagen (red) on 4th mammary gland sections from 5-week-old, pubertal WT and Adamts $18^{-/-}$littermates; $n=5$. Scale bar, $100 \mu$ m. b Representative western blot analysis on 3rd mammary glands of 5-week-old, pubertal WT and Adamts18-/- littermates; $n=4$. $\beta$-actin loading control, MW marker in red. c Dot plots showing relative protein levels of laminin, collagen I, collagen IV, and fibronectin normalized to actin in 4 pubertal WT and Adamts $18^{-/-}$littermates. Paired Student $t$-test, two-tailed; ${ }^{\star \star} p<0.01$. d, Fluorescent micrographs showing IF on 4th mammary gland sections from 5-week-old, pubertal WT and Adamts18-/littermates for laminin, collagens I and IV as well as fibronectin (green) and DAPI nuclear stain (blue), $n=3$. Arrows point to ECM density around TEBs or ducts; scale bar, $100 \mu \mathrm{m}$. e Representative western blot analysis on 3rd mammary glands of 14 -week-old $W T$ and Adamts18- $/-$ littermates; $n=3$. $\beta$-actin loading control, MW marker in red. $\mathbf{f}$ Dot plots showing relative protein levels of laminin, collagen I, collagen IV, and fibronectin normalized to actin in 3 adult $W T$ and Adamts18 $-/$ - littermates. Paired Student $t$-test, two-tailed; n.s. not significant. $\mathbf{g}$ Dot plot showing relative transcript levels of $F n 1$ normalized to Hprt in 3rd mammary glands from 6 pairs of 5-week-old WT and Adamts18-/- littermates. Paired Student $t$-test, two-tailed, n.s. not significant.

h Representative Western blot analysis of 3 independent experiments in which fibronectin (FN)-70K was incubated with purified active Adamts18 in the presence or absence of EDTA and/or protease inhibitor (PI). Anti-FN antibody specific to the N-terminal heparin-binding domain. $\mathbf{i}$ Western blot analysis of FN1-70K incubated with ADAMTS18 overexpressing HEK-293T cells in the presence or absence of EDTA. $\mathbf{j}$ Bar graph showing levels of cleaved FN in supernatants from control transfected and Adamts18 overexpressing HEK-293T cells in 2 independent experiments.

but Itga3, Itgb4, and Itgb7 were significantly altered by $p$-value. Analysis of their expression levels by qRT-PCR at puberty in mammary glands from $W T$ and Adamts $18^{-/-}$mice showed Itga3 and Itgb4, two integrins previously implicated in mammary stem cell function 47,48 and part of laminin 5 receptors, to be significantly down modulated in the mutants (Fig. 7d).

Together these findings suggest that Adamts18 is required for activation of the Hippo pathway, which in turn induces Fgfr2 expression, activation of which is critical for stem cell function. Consistent with this scenario, the 3 Hippo target genes, Ctgf, Fgfr2, and Gata $3^{46,49}$ were reduced to $73 \%, 68 \%$ or $78 \%$ of $W T$ levels, respectively, in additional transplants in the absence of Adamts18 (Fig. 7e). Double-IF for Yap and the myoepithelial marker $\alpha$-smooth muscle actin (Sma) showed expected nuclear localization of Yap in WT myoepithelial cells (Fig. $7 \mathrm{f})^{50}$. In the contralateral Adamts $18^{-/-}$.EGFP ${ }^{+}$epithelia the signal intensity of Yap was decreased in myoepithelial cells (Fig. 7f). Quantitative image analysis revealed that the mean nuclear intensity of the Yap staining in the mutant epithelium was $58 \%$ of the contralateral WT.EGFP ${ }^{+}$transplanted glands (Fig. $7 \mathrm{~g}$ ).

To further support our claim that BM modulation by Adamts18 involves the Yap/Taz signaling pathway, we assessed the expression levels of downstream targets, Cited-1, Ctgf, Fgfr2, Gata3 in pubertal WT, Col18a1-/-, Adamts18 ${ }^{-/-}$, and DKO mice. Additionally, we assessed the expression levels of Itga3 and Itgb4 altered in Adamts $18^{-/}$mice. In line with our previous findings (Fig. 7h), we found the Yap/Taz targets to be significantly down modulated in pubertal Adamts $18^{-/-}$and the DKO. Col18a1 $1^{-/}$glands displayed downmodulation in Adamts18, Cited-1, and Ctgf. This suggests that modulation of the BM composition by Adamts 18 leads to activation of Yap/Taz signaling with increased Fgfr2 expression and signaling which results in stem cell activation.

ADAMTS18 in the human breast. Our data indicate that Adamts 18 translates the hormonal stimuli received by luminal cells into activation of stem cells via changes to the BM in the mouse mammary gland. To assess whether this signaling axis may also operate in the human breast, we generated a polyclonal antibody to ADAMTS18 and validated it on MCF10A overexpressing V5 tagged human ADAMTS18 with or without a short hairpin RNA (shRNA) to knock down overexpressed ADAMTS18 (Supplementary Fig. 4). IHC of reduction mammoplasty sections showed ADAMTS18 expression was not detected in the CK7+ luminal compartment, but in myoepithelial cells identified by p63 immunostaining (Fig. 8a) as observed for the transcripts in the mouse.

To test whether expression of ADAMTS18 transcripts in human breast epithelial cells is similarly controlled by PR signaling, we humanized mouse mammary glands ${ }^{51}$. Human breast epithelial cells isolated from 4 different reduction mammoplasty specimens were infected with lentiviruses expressing luciferase-GFP and injected into the milk ducts of immune-compromised NOD scid gamma females ${ }^{51}$ (Fig. 8b). Once photon flux reached $10^{7}$ per gland, the mice received subcutaneous pellets containing either vehicle, 20 , or $50 \mathrm{mg}$ progesterone (Fig. $8 \mathrm{c}$, d). The hormonecontaining pellets resulted in 7.2- and 19.7-fold increased plasma progesterone levels, respectively (Fig. 8c); Adamts18 transcript levels were 1.8- and 2.3-fold higher than in noninjected mammary glands from the progesterone-treated mice, respectively, indicating that prolonged progesterone exposure results in increased Adamts18 transcript levels in the mouse mammary glands (Fig. 8d). Next, we dissociated the xenografted glands to single cells and enriched for the human cells by depleting mouse cells with immunomagnetic beads. The xenografted cells from 4 different patients exposed to progesterone showed increased expression of ADAMTS18 compared to control cells with an average 3-fold increase (Fig. 8e). Thus, the progesterone/ ADAMTS18 axis is conserved between mice and humans.

\section{Discussion}

Here, we have addressed the longstanding puzzle of how epithelial ER and PR signaling connect to ECM changes that accompany both normal breast development and breast carcinogenesis. We show that the gene encoding Adamts18 is expressed in the myoepithelium downstream of Wnt4 secretion induced by ER/PR signaling luminal sensor cells (Fig. 9). The myoepithelial cells respond by canonical Wnt signaling activation and link luminal hormone receptor signaling to stromal changes with functional consequences. Our finding that altered BM composition affects MaSCs shows that the BM is a central part of the stem cell niche and a critical determinant of stem cell function.

The precise nature of the BM and interstitial ECM changes that alter signaling remain to be determined. Numerous factors, such as tissue stiffness and growth factor availability, directly or indirectly controlled by Adamts 18 may be critical. The observed changes in the abundance of collagen I, collagen IV, laminin, fibronectin, and glycoproteins, like collagen XVIII, may be secondary to the reduced fibronectin clearance but Adamts18 may also be directly involved in their processing; other family members have glycoprotein substrates 14 .

Increased laminin expression was also observed in Adamts $18^{-/-}$ adipose tissue ${ }^{52}$ and embryonic brains ${ }^{53}$ with effects on early adipocyte differentiation and spine and synapse formation. A detailed analysis of kidney and lung development in WT and Adamts $18^{-/-}$ mice revealed that expression of the enzyme by branching tips is important for branching and organ size $e^{18}$.

We identified enhanced Yap/Taz nuclear localization and increased Fgfr2 signaling as potential mechanisms underlying 
a

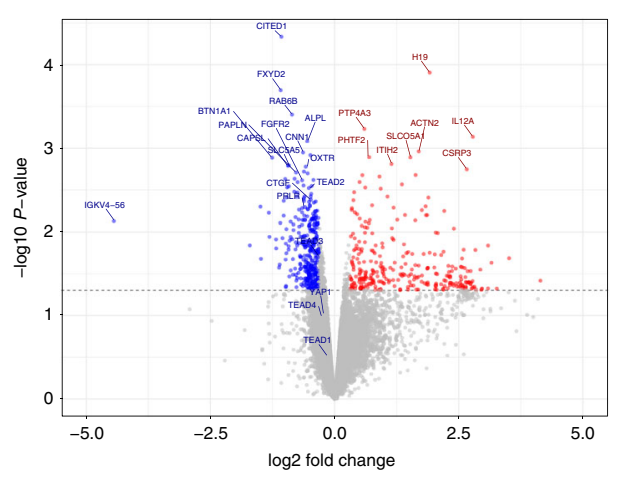

C

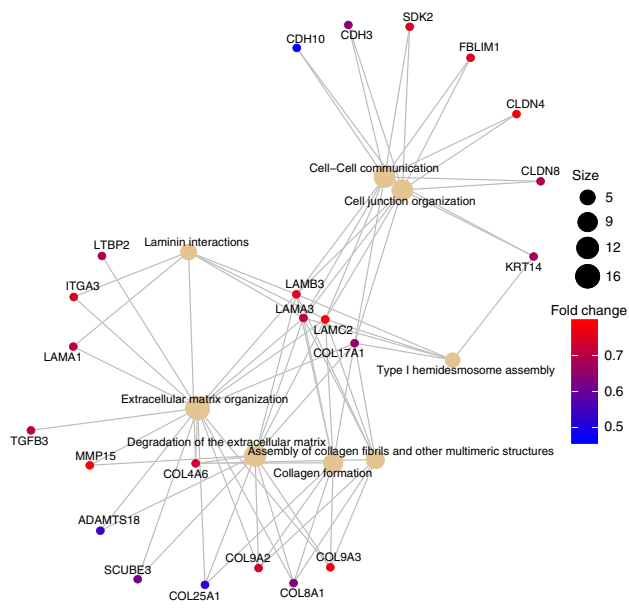

b

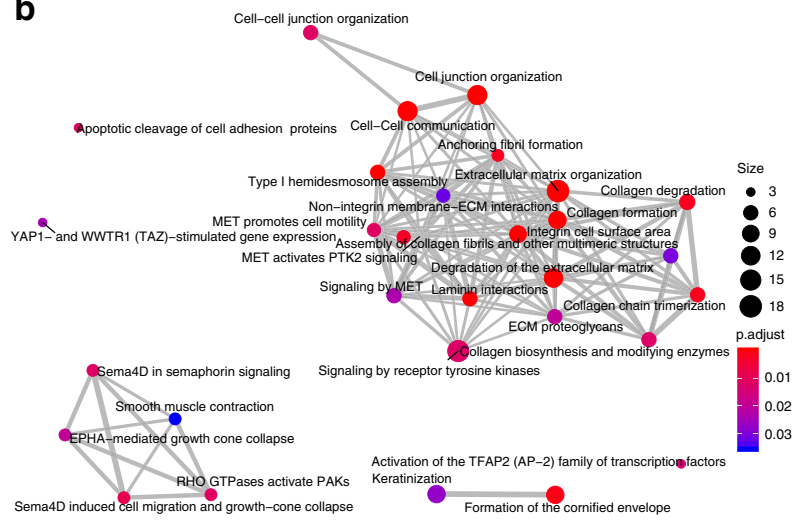

d

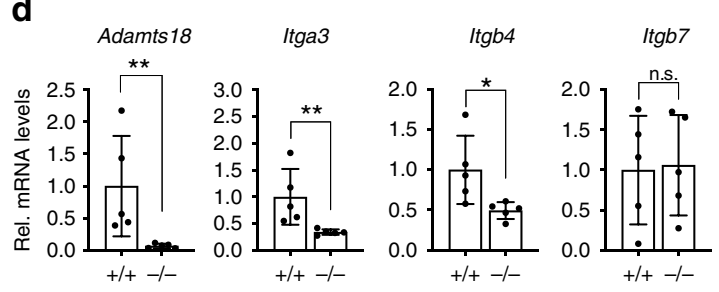

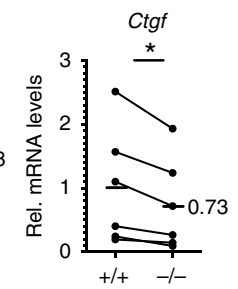

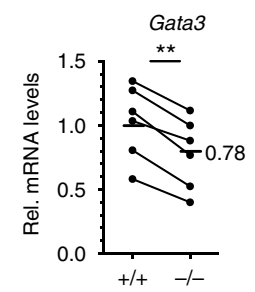

f
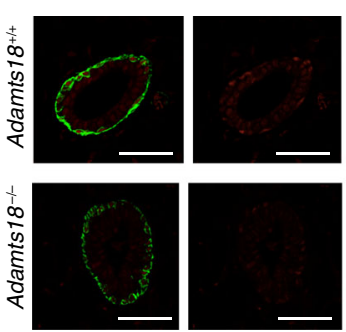
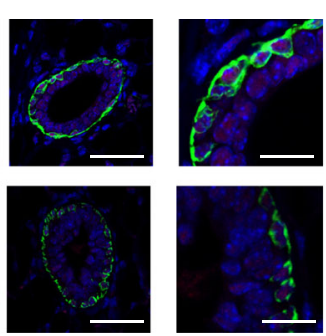

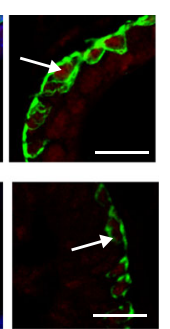

g

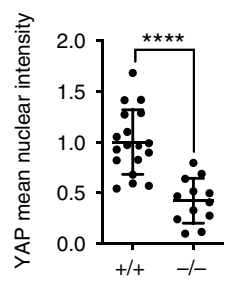

h
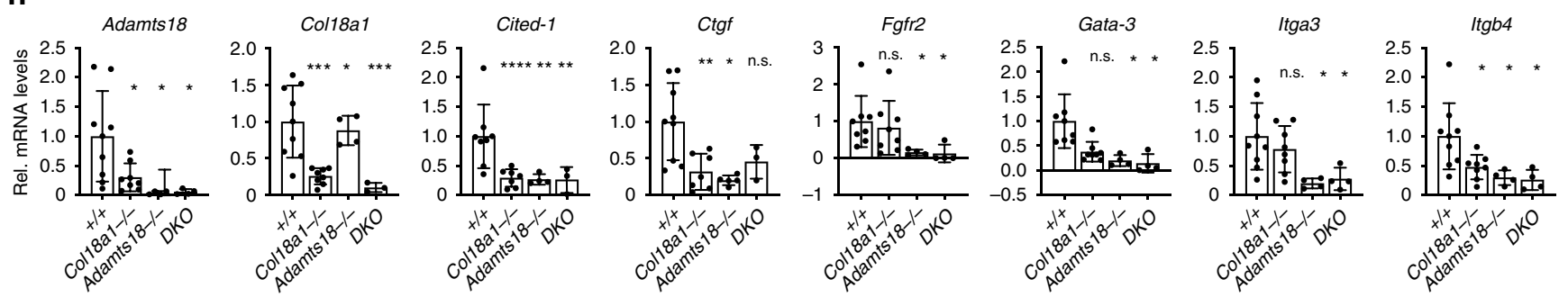

stem cell activation downstream of Adamts18 activity (Fig. 9). Whether Yap/Taz activation is central to increased Fgfr2 signaling and/or whether biochemical changes in the BM result in increased ligand availability was not addressed in our study. Yap/Taz signaling is typically activated by extracellular cues such as increased stiffness. Our gene expression analysis did not provide direct indications for this; whether the increased expression of musclerelated genes may also impinge on Yap/Taz or whether another stiffness independent mechanism ${ }^{54}$ is important, remains to be explored. We speculate that Adamts18-induced modifications of the ECM affect integrin-mediated, F-actin dependent cell-ECM adhesion and contraction, which promote cellular mechanical tension and Yap/Taz activation ${ }^{55}$. As such, the progesterone/ Wnt4/Adamts18 axis provides an entry point for further studies of epithelial-BM interactions.

The regulatory axis we identified genetically in the mouse mammary gland likely operates in the human breast with implication for breast cancer prevention and treatment. Exposure to progesterone as it occurs recurrently during menstrual cycles has been shown to induce WNT4 expression ${ }^{56,57}$ and can increase 
Fig. 7 Adamts18 impinges on transcription and regulates cell signaling. a Volcano plot showing genes, which are differentially expressed between contralateral glands transplanted with Adamts $18^{-/-}$and WT epithelia; $n=3$, Kolmogorov-Smirnov test, all highlighted genes have $p$-values $<0.05$. Genes with $\log 2(\mathrm{FC})>0.5$ in red and $\log 2 \mathrm{FC}<0.5$ in blue. Names of selected genes are indicated. $\mathbf{b}$ Enrichment map plot of Reactome pathway analysis (ReactomePA) on genes downregulated in 3 pairs of contralateral glands engrafted with WT and Adamts $18^{-/-}$epithelia in 3 independent experiments with 3 different donors. c CNE plot of ReactomePA of genes down regulated in contralateral glands transplanted with WT and Adamts18-/- epithelia. d Bar graphs showing relative transcript levels of Adamts18, Itga3, Itgb4, and Itgbt, normalized to Hprt in 5 pubertal host mice bearing contralateral transplants of WT and Adamts18-/- epithelia. Data represent mean \pm SD. Unpaired Student $t$-test, two-tailed. e Bar graphs showing relative transcript levels of Fgfr2, Ctgf, and Gata3 normalized to Hprt in contralateral glands transplanted with WT and Adamts $18^{-/-}$epithelia, $n=6$. f Representative IF for Sma (green) and YAP (red) counterstained with DAPI (blue) of 4th mammary gland sections from 5-week-old WT and Adamts $18^{-/-}$littermates; $n=3$. Arrows indicate YAP positive nuclei of myoepithelial cells. $\mathbf{g}$ Dot plot showing quantification of relative mean intensity of nuclear YAP detected in myoepithelial cells of 5-weekold WT and Adamts18-/- littermates; $n=3$. Each point represents an individual TEB. $\mathbf{h}$ Bar graphs showing relative transcript levels of Adamts18, Col18a1, Cited-1, Ctgf, Fgfr2, Gata-3, Itga3, and Itgb4, normalized to Hprt in pubertal WT, Col18a1-/-, Adamts18 ${ }^{-/-}$, and DKO; $n=9,8,4$, and 4, respectively. Data represent mean $\pm \mathrm{SD}$, one-way ANOVA. ${ }^{\star} p<0.05 ;{ }^{\star \star} p<0.01 ;{ }^{\star \star \star} p<0.001 ;{ }^{\star \star \star \star} p<0.0001$, n.s. not significant.

a

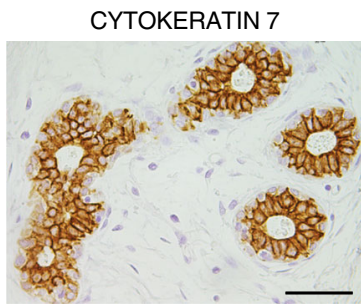

b

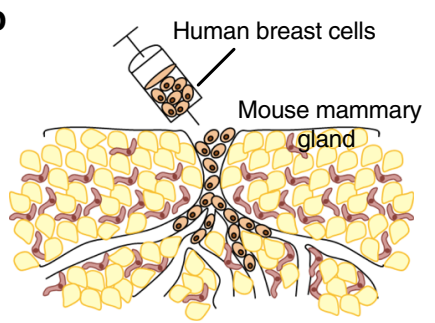

$\triangle N P 63$

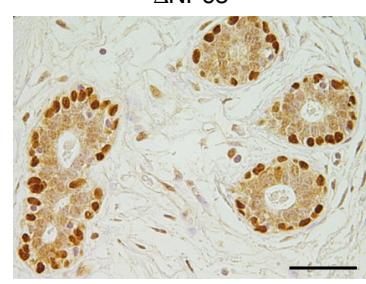

C

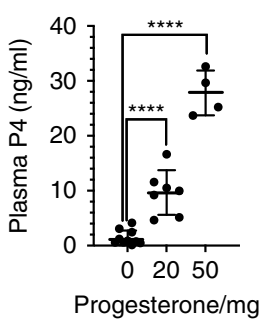

d

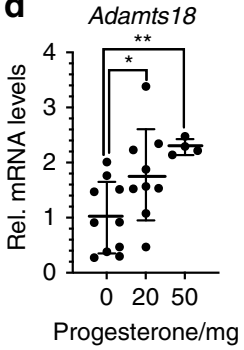

ADAMTS18

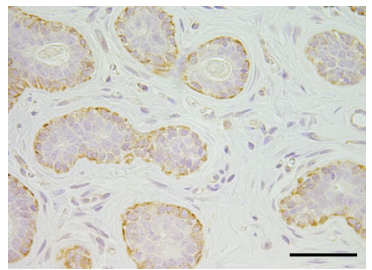

e ADAMTS18

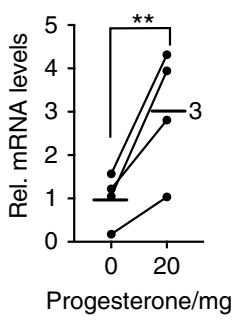

Fig. 8 ADAMTS18 expression and distribution in the human breast epithelium. a Representative micrographs of normal human breast tissue from 5 different women stained with luminal cell marker CK7, myoepithelial cell marker P63 and anti-ADAMTS18, counterstained with hematoxylin. Scale bar, $100 \mu \mathrm{m}$. b Experimental scheme: dissociated human breast epithelial cells from reduction mammoplasties were injected via the teat into the milk duct system of NSG female mice and establish themselves there. c LC/MS measured serum progesterone levels in mice 60 days after implantation with pellets containing vehicle, 20 or $50 \mathrm{mg}$ progesterone. Data represent mean \pm SD from $n=10$ (vehicle), $n=7$ (20 mg), and $n=4$ ( $50 \mathrm{mg}$ ); one-way ANOVA. d Dot plot showing Adamts 18 transcript levels as measured by semi qRT-PCR normalized to the geometric mean of Hprt and Gapdh in mammary glands from mice that were subcutaneously engrafted with pellets containing either vehicle (0) or 20 or $50 \mathrm{mg}$ progesterone for 60 days. Data represent mean \pm SD from $n=10$ (vehicle), $n=7$ (20 mg), and $n=4$ (50 mg); one-way ANOVA. e Dot plot showing relative ADAMTS18 transcript levels normalized to GAPDH in glands xenografted with human breast epithelial cells from 4 mammoplasty specimens. Recipient mice were either implanted with vehicle- or 20 mg progesterone-containing pellets. Paired $t$-test, two-tailed. ${ }^{\star} p<0.05 ;{ }^{\star \star} p<0.01 ;{ }^{* \star} p<0.001 ;{ }^{\star \star \star \star} p<0.0001$.

ADAMTS18 expression, as we show here. The resulting BM/ECM remodeling may contribute to the increased breast cancer risk associated with recurrent menstrual cycles. Furthermore, the increased risk of postmenopausal women exposed to combined hormone replacement therapy with ethinyl estradiol and progestins may, at least in part, be attributable to increased stem cell divisions and stromal alterations ${ }^{58,59}$ elicited by ADAMTS18.

Premenopausal patients with in situ carcinoma or early stage invasive disease, as well as women with high risk for breast cancer, may benefit from a preventive treatment that interferes with PR signaling or its downstream effectors. Blocking progesterone action, while possibly protective for the breast, will have many side effects as its actions are complex and affect many organs. Similarly, targeting downstream Wnt signaling has potential side effects because this signaling pathway is physiologically important for stem cells in many tissues. Based on the mouse model, ADAMTS18 is important for development of specific organs but it does not appear to have an essential function in adult mice ${ }^{31}$. Furthermore, in its extracellular location ADAMTS18 makes it an excellent target for antibody-mediated therapy. As such, targeting ADAMTS18 appears as a feasible strategy for primary and secondary prevention unlikely to elicit major side effects.

\section{Methods}

Mice. All mice were maintained and handled according to Swiss guidelines for animal safety and experiments were performed in accordance with protocols approved by the Service de la Consommation et des Affaires Vétérinaires of Canton de Vaud, Switzerland, with a 12-h-light-12-h-dark cycle, controlled temperature and food and water ad libitum. 129SV/C57BL6, $\mathrm{mT} / \mathrm{mG}^{60}$, and NOD.Cg-Prkdcscid Il2rgtm $1 \mathrm{Wjl} / \mathrm{SzJ}$ (NSG) mice were purchased from Jackson Laboratories and C57BL/6JOlaHsd mice from Harlan Laboratories. Adamts $18^{-1-31}$, Col18a1 $^{+/-61}$ MMTV::Cre (lineA $)^{62}, W_{n t} 4^{+/-63}, W_{n t 4^{\mathrm{fl} / \mathrm{fl}}} 64$, and $\operatorname{Tg}(\text { Act-EGFP })^{32}$ mice were maintained in C57BL/6JOlaHsd background.

Patient sample processing. The cantonal ethics committee approved the study $(183 / 10)$. Breast tissue was obtained from women undergoing reduction mammoplasties with no previous history of breast cancer. All human subjects provided 


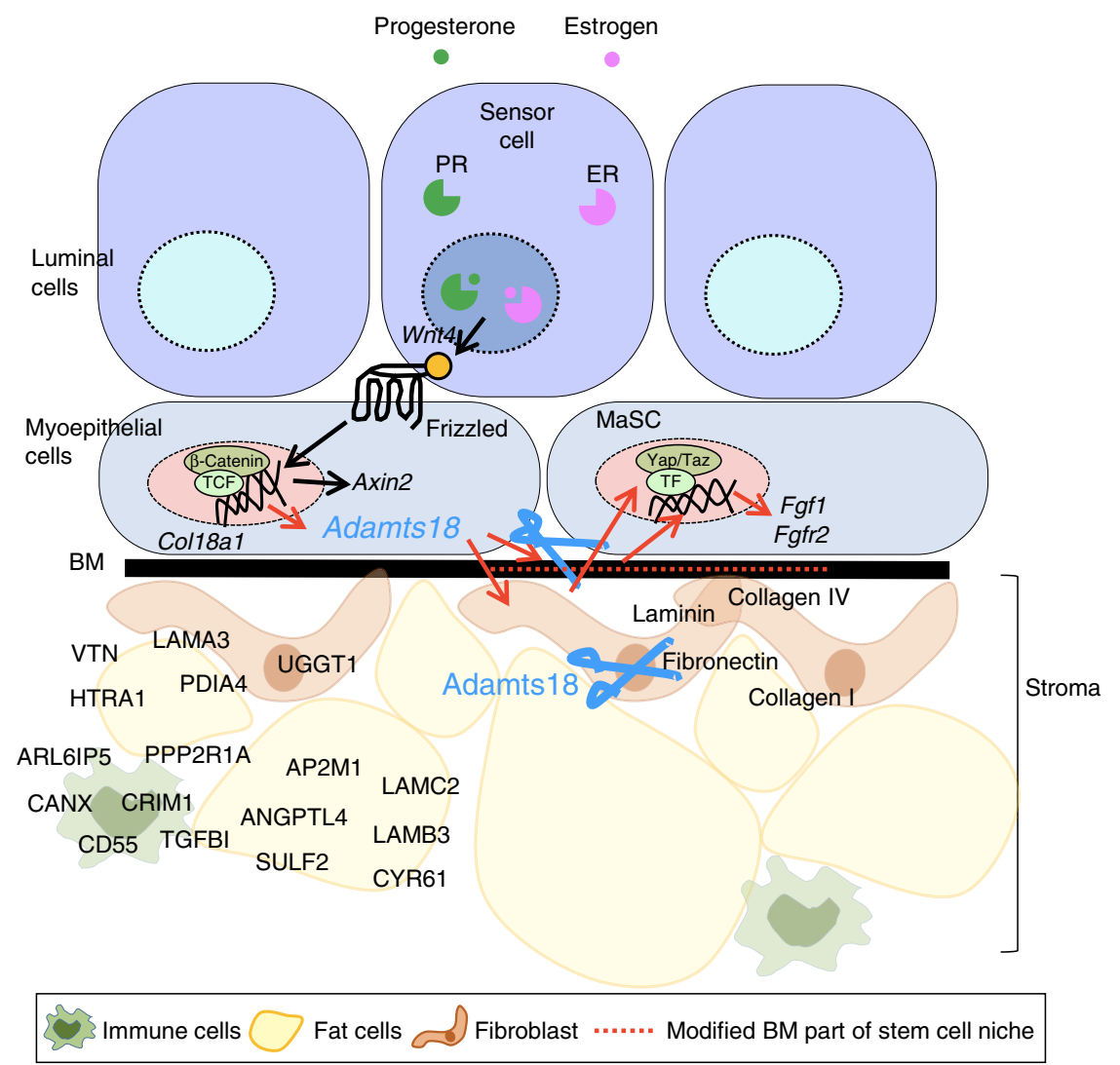

Fig. 9 Working model of Adamts18 as a modulator of mammary gland development. A schematic representation of the mammary acinar wall shows the spatial relationship between luminal cells, myoepithelial cells, BM and the surrounding interstitial ECM. Estrogen and progesterone induce Adamts18 production in myoepithelial cells via Wnt4-stimulated canonical Wnt signaling. Adamts18 remodels the BM and/or interstitial ECM, as part of the stem cell niche to ensure optimal stem cell regenerative capacity. Loss of Adamts18 alters the stem cell niche and decreases mammary epithelial regenerative potential as its essential ECM modulatory function is abrogated.

informed consent for use of tissue samples in research. Samples were examined by the pathologist to be free of malignancy.

Histology. Inguinal mammary glands were fixed in $4 \%$ PFA in phosphate-buffered saline (PBS, pH 7.2) overnight at $4^{\circ} \mathrm{C}$, embedded in paraffin and cut into $4 \mu \mathrm{m}$ sections. Hematoxylin and eosin or sirius red staining were performed according to standard protocols. For immunostaining, sections were de-waxed, rehydrated and subjected to antigen retrieval with $10 \mathrm{mM}$ citrate buffer, $\mathrm{pH} 6.0$ for 20 min at $95^{\circ} \mathrm{C}$. Sections were counterstained with Mayer's hematoxylin. For fluorescence microscopy, nuclei were counterstained with DAPI (Sigma). IF images were acquired on Leica DM 4000 B LED with Leica DFC 7000T camera and on Zeiss LSM700 confocal microscope for colocalizations. Primary antibodies A rabbit antiADAMTS18 antibody was raised against the peptide GQYKYPDKLPGQIYDA corresponding to ADAMTS18 sequence 502-516 aa (Eurogentec) an epitope that is conserved between human and mouse proteins, absent from other proteins and selected for high antigenic potential. The percentage of ER+ and PR + cells were quantified using ImageJ, the percentage of SMA+ cells with QuPath software. Antibody list can be found in Supplementary Table 4.

RNA in situ Hybridization. Adamts18 ISH was performed using RNAScope (Advanced Cell Diagnostics, Newark, CA) following the manufacturer's protocol. Briefly, $4 \mu \mathrm{m}$ sections were deparaffinized and hybridized to a mouse Adamts 18 probe set (452251; Advanced Cell Diagnostics) using a HybEZ oven (Advanced Cell Diagnostics) and the RNAScope 2.5 HD Detection Reagent Kit (322360; Advanced Cell Diagnostics) and stained with anti-SMA after the RNAScope procedure.

Transplantation. Fat pads were transplanted onto the abdominal muscle wall of adult $W T$ females $^{29}$. Single cell suspensions of mammary epithelial cells in $20 \%$ matrigel were injected and $1 \mathrm{~mm}^{3}$ of epithelial fragments were transplanted to cleared fat pads. Intraductal injection of human breast epithelial cells was performed via cleaved teat.
Mammary gland wholemounts. Mammary gland whole-mounts were performed as described ${ }^{65}$, and stereomicrographs were acquired with a LEICA MZ FLIII stereomicroscope and Leica MC170 HD. Fluorescence stereomicrographs were acquired on a LEICA M205FA with a Leica DFC 340FX camera. Fat pad filling and branching points were determined using ImageJ software.

Single cell preparation. Reduction mammoplasty microstructures were incubated with $1 \%$ collagenase A (Roche, final concentration of $1.0 \mathrm{mg} / \mathrm{ml}$ ) in (DMEM)/F12 Dulbecco's modified Eagle's medium containing 1\% penicillin/streptomycin (cat. 15070-063; Thermo Fisher Scientific) and 1\% fungizone (cat. 15290-018; Thermo Fisher), overnight at $37^{\circ} \mathrm{C}$. Cells were dissociated to single cells with $0.25 \%$ trypsinEDTA (Gibco, 15400), resuspended with red cell blood lysis buffer, and passed through $40 \mu \mathrm{m}$ cell strainer. To isolate human cells from humanized mammary glands, single cells were incubated with mouse cell depletion cocktail (Miltenyi Biotec, 130-104-694) and passed through LS columns (130-042-401) on MACS separator according to manufacturer's protocol (Miltenyi Biotec).

Hormone measurements. Progesterone hormone levels in the plasma were measured using LC-MS (Q-Exactive, ThermoFisher Scientific) ${ }^{66}$.

Fluorescence activated cell sorting. Single cell suspensions of mammary glands from 15- to 25 -week-old virgin females were processed as described ${ }^{34}$ and sorted on a FACSAria (Becton Dickinson).

Hormone treatments. Low consistency silicon elastomer (MED-4011) two parts (part A, MP3745/E81949 and part B, MP3744/E81950) were mixed with hormone powder, incubated at $37^{\circ} \mathrm{C}$ overnight as described ${ }^{67}$, and implanted subcutaneously. Three-week-old mice were ovariectomized and injected subcutaneously 10 days later with $17-\beta$-estradiol $5 \mathrm{ng} / \mathrm{g}$ of body weight (Sigma-Aldrich, St. Louis, MO) using $5 \mathrm{mg} / \mathrm{ml}$ in $100 \%$ ethanol stock or vehicle. Mammary glands were harvested $18 \mathrm{~h}$ after injection. 
RT-PCR. Mammary glands were homogenized with TRIzol reagent (Invitrogen), total RNA was isolated with miRNeasy Mini Kit (Qiagen), cDNA was synthesized with random $\mathrm{p}(\mathrm{dN})_{6}$ primers (Roche) and MMLV reverse transcriptase (Invitrogen). Real-time PCR analysis in triplicates was performed with SYBR Green FastMix (Quanta) reaction mix. Primers used for RT-PCR, see Supplementary Table 5.

Protein extraction and western blot. Total proteins from the 3rd mammary glands of 5- and 14-week-old WT and Adamts $18^{-/-}$littermate mice were extracted in Nonidet P-40 (NP-40) lysis buffer (2\% NP- $40,80 \mathrm{mM} \mathrm{NaCl}, 100 \mathrm{mM}$ Tris- $\mathrm{HCl}$ and $0.1 \%$ SDS) with a tissue disruptor on ice. $500 \mu \mathrm{l}$ of buffer was used for $100 \mathrm{mg}$ tissue and debris was removed by centrifugation. Transfected MCF-7 and MCF-10A were lysed with RIPA lysis buffer supplemented with protease inhibitors and protein concentration measured with a BCA kit (Pierce). Equal amounts of protein samples were subjected to SDS-PAGE on an $8 \%$ gel and electroblotted to PVDF membranes. Membranes were probed with fibronectin (Abcam ab2413), collagen IV (Abcam ab6586), collagen I (Abcam ab34710), laminin (Abcam ab30320), Lamin B1 (Abcam AB16048), ADAMTS18 (Eurogetech) and $\beta$-actin (Sigma mab1501) antibodies. IRDye conjugated secondary antibodies were detected with Odyssey CLx membrane scanner with Li-COR and band intensities quantified by ImageJ.

AP-MS analysis for ADAMTS18 binding proteins. MCF-10A cells were spininfected with an ADAMTS18 lentivirus containing a V5 tag or LacZ control virus. Cells were cultured to confluence in $10 \mathrm{~cm}$ dishes. Proteins were extracted with RIPA lysis buffer supplemented with protease inhibitors and protein concentration measured with a BCA kit (Pierce). ADAMTS18 was immunoprecipitated from $1 \mathrm{mg}$ of protein using anti-V5 antibody conjugated agarose beads (Sigma A7345). The immune precipitates were subjected to SDS-PAGE, the gel was stained with colloidal Coomasie blue (Biorad), bands were excised and subjected to reduction/ alkylation followed by tryptic digestion and LC-MS/MS proteomic analysis. Detected peptides were mapped against the human protein database, label-free protein quantification was performed and affinity lists were constructed in Scaffold 4 Proteomics Software using a minimum of 2 peptides to identify the proteins with a peptide false discovery rate (FDR) of $0.1 \%$ and protein FDR of $0.3 \%$.

Cloning. $\triangle$ CT-Adamts18-867aa cDNAs were amplified from cDNA library prepared from eyes and fused to FLAG-tag and $\mathrm{His}_{6}$-tag at $\mathrm{N}$-terminus by PCR and cloned into NheI and HindIII restriction sites of pcDNA3.1/Hygromycin expression vector (Invitrogen). Plasmids were purified with HighPure midiprep kit (Invitrogen).

Fibronectin cleavage. $500 \mathrm{ng}$ purified $70 \mathrm{~K}$ fibronectin (Sigma) were mixed with $50 \mathrm{ng}$ of purified $\triangle \mathrm{Ct}$-ADAMTS18 in digestion buffer $(50 \mathrm{mM}$ Tris- $\mathrm{HCl}, \mathrm{pH} 7.5$, $\left.150 \mathrm{mM} \mathrm{NaCl}, 10 \mathrm{mM} \mathrm{CaCl}, 5 \mu \mathrm{M} \mathrm{ZnCl}_{2}\right)$, incubated $24 \mathrm{~h}$ at $37^{\circ} \mathrm{C}$ in presence or absence of EDTA $(25 \mathrm{mM})$ and PI (Pierce), and analyzed by WB with ABC antibody. $24 \mathrm{~h}$ after $\triangle \mathrm{Ct}$-ADAMTS18 transfected $293 \mathrm{~T}$ cells were supplemented with $2 \mu \mathrm{g} / \mathrm{ml}$ purified $70 \mathrm{~K}$ fibronectin (Sigma) and heparin $(100 \mu \mathrm{g} / \mathrm{ml})$.

Bioinformatic analysis: For details of RNA-seq and microarray analyses see Supplementary Methods.

Statistics. Prism 6 software (GraphPad) used for statistical analyses and the statistical tests with their reported $p$-values are indicated in each figure.

Reporting Summary. Further information on research design is available in the Nature Research Reporting Summary linked to this article.

\section{Data availability}

The authors declare that all data supporting the findings of this study are available within the article and its Supplementary Information files or from the corresponding author upon reasonable request. The datasets generated and analyzed during the current study have been deposited in the GEO database under the accession code: GSE145717, for microarray data and GSE145680 for RNA sequencing.

Received: 19 July 2019; Accepted: 28 February 2020;

Published online: 26 March 2020

\section{References}

1. Clarke, R. B., Howell, A., Potten, C. S. \& Anderson, E. Dissociation between steroid receptor expression and cell proliferation in the human breast. Cancer Res. 57, 4987-4991 (1997).

2. Brisken, C. \& Duss, S. Stem cells and the stem cell niche in the breast: an integrated hormonal and developmental perspective. Stem Cell Rev. 3, 147-156 (2007).
3. Beleut, M. et al. Two distinct mechanisms underlie progesterone-induced proliferation in the mammary gland. Proc. Natl. Acad. Sci. USA 107, 2989-2994 (2010)

4. Ciarloni, L., Mallepell, S. \& Brisken, C. Amphiregulin is an essential mediator of estrogen receptor function in mammary gland development. Proc. Natl. Acad. Sci. USA 104, 5455-5460 (2007).

5. Shiah, Y.-J. et al. A progesterone-CXCR4 axis controls mammary progenitor cell fate in the adult gland. Stem Cell Rep. 4, 313-322 (2015).

6. Rajaram, R. D. et al. Progesterone and Wnt4 control mammary stem cells via myoepithelial crosstalk. EMBO J. 34, 641-652 (2015).

7. Visvader, J. E. \& Stingl, J. Mammary stem cells and the differentiation hierarchy: current status and perspectives. Genes Dev. 28, 1143-1158 (2014).

8. Bruno, R. D. \& Smith, G. H. Reprogramming non-mammary and cancer cells in the developing mouse mammary gland. Semin. Cell Dev. Biol. 23, 591-598 (2012).

9. Bruno, R. D. et al. Mammary extracellular matrix directs differentiation of testicular and embryonic stem cells to form functional mammary glands in vivo. Sci. Rep. 7, 1-10 (2017).

10. Zhao, C. et al. Stromal Gli2 activity coordinates a niche signaling program for mammary epithelial stem cells. Science 356, eaal3485 (2017).

11. Alowami, S., Troup, S., Al-Haddad, S., Kirkpatrick, I. \& Watson, P. H. Mammographic density is related to stroma and stromal proteoglycan expression. Breast Cancer Res. 5, 129-135 (2003).

12. Byrne, C. et al. Mammographic density change with estrogen and progestin therapy and breast cancer risk. J. Natl. Cancer Inst. 109, 1-7 (2017).

13. Kuno, K. et al. Molecular cloning of a gene encoding a new type of metalloproteinase-disintegrin family protein with thrombospondin motifs as an inflammation associated gene. J. Biol. Chem. 272, 556-562 (1997).

14. Apte, S. S. A disintegrin-like and metalloprotease (reprolysin-type) with thrombospondin type 1 motif (ADAMTS) superfamily: functions and mechanisms. J. Biol. Chem. 284, 31493-31497 (2009).

15. Kelwick, R., Desanlis, I., Wheeler, G. N. \& Edwards, D. R. The ADAMTS (A Disintegrin and Metalloproteinase with Thrombospondin motifs) family. Genome Biol. 16, 113 (2015).

16. Hurskainen, T. L., Hirohata, S., Seldin, M. F. \& Apte, S. S. ADAM-TS5, ADAM-TS6, and ADAM-TS7, novel members of a new family of zinc metalloproteases: general features and genomic distribution of the ADAM-TS family. J. Biol. Chem. 274, 25555-25563 (1999).

17. Zheng, X. et al. Structure of von Willebrand factor-cleaving protease (ADAMTS13), a metalloprotease involved in thrombotic thrombocytopenic purpura. J. Biol. Chem. 276, 41059-41063 (2001).

18. Rutledge, E. A., Parvez, R. K., Short, K. M., Smyth, I. M. \& McMahon, A. P. Morphogenesis of the kidney and lung requires branch-tip directed activity of the Adamts18 metalloprotease. Dev. Biol. 454, 156-169 (2019).

19. Abdul-Majeed, S., Mell, B., Nauli, S. M. \& Joe, B. Cryptorchidism and infertility in rats with targeted disruption of the Adamts16 locus. PLOS ONE 9 , e100967 (2014)

20. Gopalakrishnan, K. et al. Targeted disruption of Adamts16 gene in a rat genetic model of hypertension. Proc. Natl. Acad. Sci. USA 109, 20555-20559 (2012).

21. Schnellmann, R., Sack, R., Hess, D., Annis, D. S. \& Mosher, D. F. A selective extracellular matrix proteomics approach identifies fibronectin proteolysis by a disintegrin-like and metalloprotease domain with thrombospondin type 1 motifs (ADAMTS16) and its impact on spheroid morphogenesis. Mol. Cell. Proteom. 17, 1410-1425 (2018).

22. Fernandez-Valdivia, R. et al. Transcriptional response of the murine mammary gland to acute progesterone exposure. Endocrinology 149, 6236-6250 (2008)

23. Lain, A. R., Creighton, C. J. \& Conneely, O. M. research resource: progesterone receptor targetome underlying mammary gland branching morphogenesis. Mol. Endocrinol. 27, 1743-1761 (2013).

24. Kendrick, H. et al. Transcriptome analysis of mammary epithelial subpopulations identifies novel determinants of lineage commitment and cell fate. BMC Genomics 9, 591 (2008).

25. Pal, B. et al. Construction of developmental lineage relationships in the mouse mammary gland by single-cell RNA profiling. Nat. Commun. 8, 1627 (2017).

26. Brisken, C. et al. Essential function of Wnt- 4 in mammary gland development downstream of progesterone signaling. Genes Dev. 14, 650-654 (2000).

27. Chen, C. et al. Genome-wide ChIP-seq analysis of TCF4 binding regions in colorectal cancer cells. Int. J. Clin. Exp. Med. 7, 4253 (2014).

28. Tsukamoto, A. S., Grosschedl, R., Guzman, R. C. \& Varmus', H. E. Expression of the int-1 gene in transgenic mice is associated with mammary gland hyperplasia and adenocarcinomas in male and female mice. Cell 55, 619-625 (1988).

29. Brisken, C. et al. A paracrine role for the epithelial progesterone receptor in mammary gland development. Proc. Natl. Acad. Sci. USA 95, 5076-5081 (1998).

30. Brisken, C. et al. IGF-2 is a mediator of prolactin-induced morphogenesis in the breast. Dev. Cell 3, 877-887 (2002). 
31. Ataca, D. et al. Adamts18 deletion results in distinct developmental defects and provides a model for congenital disorders of lens, lung, and female reproductive tract development. Biol. Open 5, 1585-1594 (2016).

32. Okabe, M., Ikawa, M., Kominami, K., Nakanishi, T. \& Nishimune, Y. 'Green mice' as a source of ubiquitous green cells. FEBS Lett. 407, 313-319 (1997).

33. Mallepell, S., Krust, A., Chambon, P. \& Brisken, C. Paracrine signaling through the epithelial estrogen receptor $\alpha$ is required for proliferation and morphogenesis in the mammary gland. Proc. Natl. Acad. Sci. USA 103, 6 (2006).

34. Stingl, J. et al. Purification and unique properties of mammary epithelial stem cells. Nature 439, 993-997 (2006).

35. Hu, Y. \& Smyth, G. K. ELDA: extreme limiting dilution analysis for comparing depleted and enriched populations in stem cell and other assays. J. Immunol. Methods 347, 70-78 (2009).

36. Daniel, W., DeOme, K. B., Young, J. T., Blair, P. B. \& Faulkin, L. J. The in vivo Life Span of normal and preneoplastic mouse mammary glands: a serial transplantation study. Proc. Natl. Acad. Sci. USA 61, 53-60 (1968).

37. Yalcin-Ozuysal, Ö. et al. Antagonistic roles of Notch and p63 in controlling mammary epithelial cell fates. Cell Death Differ. 17, 1600-1612 (2010).

38. Nummela, P. et al. Transforming growth factor beta-induced (TGFBI) is an anti-adhesive protein Regulating the invasive growth of melanoma cells. Am. J. Pathol. 180, 1663-1674 (2012).

39. Marneros, A. G. \& Olsen, B. R. Physiological role of collagen XVIII and endostatin. FASEB J. 19, 716-728 (2005).

40. Filla, M. S., Dimeo, K. D., Tong, T. \& Peters, D. M. Disruption of fibronectin matrix affects type IV collagen, fibrillin and laminin deposition into extracellular matrix of human trabecular meshwork (HTM) cells. Exp. Eye Res. 165, 7-19 (2017)

41. Kadler, K. E., Hill, A. \& Canty-Laird, E. G. Collagen fibrillogenesis: fibronectin, integrins, and minor collagens as organizers and nucleators. Curr. Opin. Cell Biol. 20, 495-501 (2008).

42. Yu, G. \& He, Q.-Y. ReactomePA: an R/Bioconductor package for reactome pathway analysis and visualization. Mol. Biosyst. 12, 477-479 (2016).

43. Yu, G., Wang, L.-G., Han, Y. \& He, Q.-Y. clusterProfiler: an R package for comparing biological themes among gene clusters. OMICS 16, 284-287 (2012).

44. Lu, P., Ewald, A. J., Martin, G. R. \& Werb, Z. Genetic mosaic analysis reveals FGF receptor 2 function in terminal end buds during mammary gland branching morphogenesis. Dev. Biol. 321, 77-87 (2008).

45. Pond, A. C. et al. Fibroblast growth factor receptor signaling is essential for normal mammary gland development and stem cell function. Stem Cells 31, 178-189 (2013).

46. Rizvi, S. et al. A hippo and fibroblast growth factor receptor autocrine pathway in cholangiocarcinoma. J. Biol. Chem. 291, 8031-8047 (2016).

47. Romagnoli, M. et al. Deciphering the mammary stem cell niche: a role for laminin-binding integrins. Stem Cell Rep. 12, 831-844 (2019).

48. Li, J., Sun, H., Feltri, M. L. \& Mercurio, A. M. Integrin $\beta 4$ regulation of PTHrP underlies its contribution to mammary gland development. Dev. Biol. 407, 313-320 (2015).

49. Shi, P., Feng, J. \& Chen, C. Hippo pathway in mammary gland development and breast cancer. Acta Biochim. et. Biophys. Sin. 47, 53-59 (2015).

50. Vlug, E. J. et al. Nuclear localization of the transcriptional coactivator YAP is associated with invasive lobular breast cancer. Cell. Oncol. 36, 375-384 (2013).

51. Sflomos, G. et al. A preclinical model for ERa-positive breast cancer points to the epithelial microenvironment as determinant of luminal phenotype and hormone response. Cancer Cell 29, 407-422 (2016).

52. Zhu, R. et al. A disintegrin and metalloproteinase with thrombospondin motifs 18 deficiency leads to visceral adiposity and associated metabolic syndrome in mice. Am. J. Pathol. 188, 461-473 (2018).

53. Zhu, R. et al. ADAMTS18 deficiency affects neuronal morphogenesis and reduces the levels of depression-like behaviors in mice. Neuroscience 399 , 53-64 (2019).

54. Lee, J. Y. et al. YAP-independent mechanotransduction drives breast cancer progression. Nat. Commun. 10, 1848 (2019).

55. Dupont, S. et al. Role of YAP/TAZ in mechanotransduction. Nature 474, 179-183 (2011).

56. Pardo, I. et al. Next-generation transcriptome sequencing of the premenopausal breast epithelium using specimens from a normal human breast tissue bank. Breast Cancer Res. 16, 26 (2014).

57. Tanos, T. et al. Progesterone/RANKL is a major regulatory axis in the human breast. Sci. Transl. Med. 5, 182ra55-182ra55 (2013).

58. MacMahon, B. et al. Age at first birth and breast cancer risk. Bull. World Health Organ. 43, 209 (1970).

59. Ross, R. K., Wan, P. C. \& Pike, M. C. Effect of hormone replacement therapy on breast cancer risk: estrogen versus estrogen plus progestin. J. Natl. Cancer Inst. 92, 5 (2000).
60. Muzumdar, M. D., Tasic, B., Miyamichi, K., Li, L. \& Luo, L. A global doublefluorescent Cre reporter mouse. Genesis 45, 593-605 (2007).

61. Fukai, N. Lack of collagen XVIII/endostatin results in eye abnormalities. EMBO J. 21, 1535-1544 (2002)

62. Wagner, K. U. et al. Cre-mediated gene deletion in the mammary gland. Nucleic Acids Res. 25, 4323-4330 (1997).

63. Stark, K., Vainio, S., Vassileva, G. \& McMahon, A. P. Epithelial transformation of metanephric mesenchyme in the developing kidney regulated by Wnt-4. Nature 372, 679-683 (1994).

64. Shan, J., Jokela, T., Peltoketo, H. \& Vainio, S. Generation of an allele to inactivate Wnt4 gene function conditionally in the mouse. Genesis 47, 782-788 (2009)

65. Ayyanan, A. et al. Perinatal exposure to bisphenol A increases adult mammary gland progesterone response and cell number. Mol. Endocrinol. 25, 1915-1923 (2011).

66. Cagnet, $\mathrm{S}$. et al. Oestrogen receptor a AF-1 and AF-2 domains have cell population-specific functions in the mammary epithelium. Nature Commun. 9, 4723 (2018).

67. Duss, S. et al. An oestrogen-dependent model of breast cancer created by transformation of normal human mammary epithelial cells. Breast Cancer Res. 9, 38 (2007)

\section{Acknowledgements}

We thank D. Buric and J. Dubail for advice and technical assistance, J. Dessimoz at the EPFL histology core facility, O. Burri and A. Seitz at the EPFL bioimaging and optics platform (BIOP), R. Guiet at the EPFL flow cytometry core facility (FCCF), and L. Tauzin and A. Mozes and, B. Mangeat at the EPFL gene expression core facility (GECF) for technical assistance. Col18a1 $1^{-1-}$ mice were kindly provided by Bjorn R. Olsen, Harvard Medical School. D.A. and M.S. received funding from the Swiss Cancer Ligue KFS-370108-2015, SNF 31003A_162550/1 Hormonal and cell signaling control of mammary gland morphogenesis: The role of Adamts 18 in epithelial-basal membrane interactions that control the stem cell function downstream of progesterone receptor signaling, and SNF 31003A_141248 Hormonal and cell signaling control of mammary gland morphogenesis: ER/PR and Notch signaling interactions, P.A, C.C, C.L, R.J. SNF 31003A_162550/1, and R.R. from NCCR-Oncology $1 \mathrm{~A} 1$ and SNF FN-MHV.

\section{Author contributions}

Investigation: D.A., P.A., C.C., C.L., M.B., M.S., M.C., R.R., and T.M.; Bioinformatic analysis: R.J., G.A., and P.B.; Writing: D.A., P.A., S.A., and C.B; Funding acquisition: C.B.

\section{Competing interests}

The authors declare no competing interests.

\section{Additional information}

Supplementary information is available for this paper at https://doi.org/10.1038/s41467020-15357-y.

Correspondence and requests for materials should be addressed to C.B.

Peer review information Nature Communications thanks the anonymous reviewer(s) for their contribution to the peer review of this work. Peer reviewer reports are available.

Reprints and permission information is available at http://www.nature.com/reprints

Publisher's note Springer Nature remains neutral with regard to jurisdictional claims in published maps and institutional affiliations.

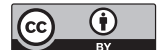

Open Access This article is licensed under a Creative Commons Attribution 4.0 International License, which permits use, sharing, adaptation, distribution and reproduction in any medium or format, as long as you give appropriate credit to the original author(s) and the source, provide a link to the Creative Commons license, and indicate if changes were made. The images or other third party material in this article are included in the article's Creative Commons license, unless indicated otherwise in a credit line to the material. If material is not included in the article's Creative Commons license and your intended use is not permitted by statutory regulation or exceeds the permitted use, you will need to obtain permission directly from the copyright holder. To view a copy of this license, visit http://creativecommons.org/ licenses/by/4.0/.

(C) The Author(s) 2020 